\title{
Renal epithelium regulates erythropoiesis via HIF-dependent suppression of erythropoietin
}

\author{
Navid M. Farsijani, ${ }^{1,2}$ Qingdu Liu, ${ }^{1,3}$ Hanako Kobayashi, ${ }^{1,3}$ Olena Davidoff, ${ }^{1,3}$ Feng Sha, Joachim Fandrey, ${ }^{2}$ T. Alp Ikizler, ${ }^{1,3}$ \\ Paul M. O'Connor, ${ }^{4}$ and Volker H. Haase ${ }^{1,3,5}$ \\ 'Department of Medicine, Vanderbilt University School of Medicine, Nashville, Tennessee, USA. Innstitut für Physiologie, Universität Duisburg-Essen, Universitätsklinikum Essen, Essen, Germany. \\ ${ }^{3}$ Medical and Research Services, Department of Veterans Affairs Hospital, Tennessee Valley Healthcare System, Nashville, Tennessee, USA. ${ }^{4}$ Departments of Medicine and Physiology, Georgia Regents \\ University, Augusta, Georgia, USA. ${ }^{5}$ Department of Cancer Biology and Department of Molecular Physiology and Biophysics, Vanderbilt University School of Medicine, Nashville, Tennessee, USA.
}

\begin{abstract}
The adult kidney plays a central role in erythropoiesis and is the main source of erythropoietin (EPO), an oxygen-sensitive glycoprotein that is essential for red blood cell production. Decreases of renal $\mathrm{pO}_{2}$ promote hypoxia-inducible factor 2mediated (HIF-2-mediated) induction of EPO in peritubular interstitial fibroblast-like cells, which serve as the cellular site of EPO synthesis in the kidney. It is not clear whether HIF signaling in other renal cell types also contributes to the regulation of EPO production. Here, we used a genetic approach in mice to investigate the role of renal epithelial HIF in erythropoiesis. Specifically, we found that HIF activation in the proximal nephron via induced inactivation of the von Hippel-Lindau tumor suppressor, which targets the HIF- $\alpha$ subunit for proteasomal degradation, led to rapid development of hypoproliferative anemia that was associated with a reduction in the number of EPO-producing renal interstitial cells. Moreover, suppression of renal EPO production was associated with increased glucose uptake, enhanced glycolysis, reduced mitochondrial mass, diminished $\mathrm{O}_{2}$ consumption, and elevated renal tissue $\mathrm{pO}_{2}$. Our genetic analysis suggests that tubulointerstitial cellular crosstalk modulates renal EPO production under conditions of epithelial HIF activation in the kidney.
\end{abstract}

\section{Introduction}

Renal oxygen sensing mechanisms play a key role in the regulation of erythropoiesis in adults, as the kidney is the main source of erythropoietin (EPO), an oxygen-sensitive glycoprotein hormone that is essential for normal erythropoiesis. EPO regulates rbc mass by promoting the survival of CFU-erythroid (CFU-E) cells and erythroblasts, which have not yet begun to synthesize hemoglobin $(\mathrm{Hb})(1,2)$. The induction of renal EPO synthesis represents a classic response to systemic hypoxia and results in increased rbc production, augmenting the oxygen-carrying capacity of blood when arterial $\mathrm{pO}_{2}\left(\mathrm{PaO}_{2}\right)$ is low. Failure to produce adequate amounts of EPO results in anemia, which is a common clinical feature of advanced chronic kidney disease (CKD) and is typically treated with recombinant EPO. Conversely, dysregulation of renal oxygen sensing, either by pharmacologic means or as a result of genetic mutations, can lead to inappropriately high EPO production and polycythemia (3).

The hypoxic induction of renal EPO is controlled by hypoxiainducible factor 2 (HIF-2) (4). HIF transcription factors consist of an oxygen-sensitive $\alpha$ subunit (HIF- $1 \alpha$, HIF- $2 \alpha$ [EPAS-1], and HIF-3 $\alpha$ ) and a constitutively expressed $\beta$ subunit, HIF- $\beta$, also known as the aryl-hydrocarbon receptor nuclear translocator (ARNT). HIF activity is controlled by oxygen-, iron-, and ascorbate-dependent prolyl4-hydroxylase domain-containing proteins 1-3 (PHD1-3), which use 2-oxoglutarate $(\mathrm{OG})$ as substrate for the hydroxylation of specific

Conflict of interest: The authors have declared that no conflict of interest exists. Submitted: January 2, 2014; Accepted: January 14, 2016.

Reference information: J Clin Invest. 2016;126(4):1425-1437. doi:10.1172/JCI74997. proline residues within HIF- $\alpha$. Under normoxia, HIF- $\alpha$ is hydroxylated and binds to the von Hippel-Lindau-E3 (VHL-E3) ubiquitin ligase complex, which subsequently leads to its proteasomal degradation (5). Under hypoxic conditions, however, HIF prolyl-4-hydroxylation is inhibited and HIF- $\alpha$ is no longer degraded and translocates to the nucleus, where heterodimerization with HIF- $\beta$ /ARNT leads to transactivation of HIF target genes.

EPO synthesis in the kidney is dependent on tissue $\mathrm{pO}_{2}$ $\left(\mathrm{PtO}_{2}\right)$, and renal EPO production is controlled by the number of renal EPO-producing cells (REPCs), i.e., under hypoxic conditions, the number of REPCs increases proportionally to the degree of systemic hypoxia $(6,7)$. REPCs are contained within the peritubular interstitial/perivascular fibroblast population and express both pericyte markers (e.g., PDGFR- $\beta$ polypeptide and ecto-5'-nucleotidase/CD73) and neuronal markers (e.g., microtubule-associated protein 2 and neurofilament protein light polypeptide) (7-9). They reside within the peritubular interstitial space of the deep cortex and outer medulla and are characterized by dendritic cellular projections, which form direct contacts with renal tubules and capillaries (7). Thus, intercellular crosstalk is likely to affect EPO synthesis in REPCs via paracrine and other signaling mechanisms or direct cell-cell interactions (10). However, these signaling mechanisms are not well understood. The degree of renal tubular workload has previously been suggested as modulating EPO production during hypoxia, presumably through effects on renal $\mathrm{O}_{2}$ consumption. Serum EPO (sEPO) levels were diminished in rats treated with acetazolamide, but not with furosemide. Both are diuretics that decrease tubular sodium reabsorption and thus decrease renal $\mathrm{O}_{2}$ consumption 
(11). A negative feedback loop also appears to operate during ascent to high altitude, where sEPO levels decrease before a significant increase in rbc mass is observed $(12,13)$. The molecular basis of this feedback regulation is not clear.

While it is well documented that hypoxia induces EPO via HIF-2 activation in REPCs, the role of hypoxia/HIF signaling in other renal cell types in the regulation of renal EPO synthesis is unclear. Here, we used a tetracycline-inducible genetic model of pan-epithelial VHL and HIF inactivation in the kidney to investigate renal epithelial HIF in the regulation of erythropoiesis. We found that acute HIF stabilization in renal epithelial cells resulted in rapid development of anemia and suppressed the induction of EPO under hypoxic conditions. This was associated with a shift of renal metabolism toward glycolysis, a reduction in mitochondrial mass and $\mathrm{O}_{2}$ consumption, and an increase in cortical $\mathrm{PtO}_{2}$ despite the presence of anemia. Our findings highlight what we believe is a novel role for renal epithelial HIF in erythropoiesis and suggest that oxygen-sensitive tubulointerstitial intercellular crosstalk modulates EPO production in REPCs.

\section{Results}

Pan-epithelial Vhl inactivation in the kidney results in rapid development of renal anemia. In order to study the effects of constitutive HIF stabilization in renal epithelial cells, we generated a mouse model of conditional $\mathrm{Vhl}$ inactivation in which a luciferase and Cre-recombinase transgene (LC-1) is under control of a tetracycline-sensitive bidirectional promoter, which is activated by a reverse tetracycline-responsive transactivator (rtTA) under control of paired box 8 ( $\mathrm{Pax} 8$ ) gene regulatory elements (14). As previously reported, Pax8-rtTA-based Cre/loxP-mediated recombination targets renal epithelial cells along the entire nephron (Figure 1A) as well as a subset of hepatocytes (14). To achieve efficient and reproducible $V h l$ inactivation, 4-week-old $V h l^{l / f l}$ Pax 8 -rtTA LC-1 mice and their Cre-negative littermates were treated with doxycycline for 2 weeks (hereafter referred to as P8; Vhllt/fl mutants and control mice). Phenotypic analysis was carried out within 2 weeks after completion of doxycycline treatment. Genomic PCR was used to confirm efficient recombination in the kidney and liver; recombination in other organs was not detected (Figure $1 \mathrm{~B}$ and data not shown). Pax8-rtTA-mediated inactivation of $\mathrm{Vhl}$ resulted in HIF- $1 \alpha$ and HIF-2 $\alpha$ stabilization in the kidney (Figure 1B), and immunohistochemical analysis revealed strong nuclear HIF-1 $\alpha$ staining in both cortical and medullary tubular epithelial cells, whereas HIF-2 $\alpha$ was predominantly found in cortical nephron segments (Supplemental Figure 1A; supplemental material available online with this article; doi:10.1172/JCI74997DS1). The nuclei of renal interstitial cells and glomeruli remained negative for both HIF- $1 \alpha$ and HIF- $2 \alpha$, which is in line with the published expression pattern of Pax8-rtTA (14). In P8; Vhll/fl livers, HIF-2 $\alpha$ was detected in a subpopulation of hepatocytes (approximately $5 \%$ of all hepatocytes) (Supplemental Figure 1A). HIF-2 $\alpha$ stabilization in hepatocytes was associated with polycythemia (hematocrit [Hct]: $65.7 \% \pm 0.7 \%$ in $\mathrm{P} 8 ; \mathrm{Vh}^{\mathrm{t} / \mathrm{fl} l}$ mutants vs. $47.7 \% \pm 1.2 \%$ in littermate controls; $n=3$ each) (Figure 1C), which is consistent with findings from our and other laboratories $(15,16)$. Hepatic Epo transcript levels were strongly increased in P8; Vhll/fl mutant mice, while renal Epo mRNA levels were suppressed, indicating that the kidneys of P8; Vhl $l^{l / f l}$ mutant mice responded physiologically to the presence of polycythemia and that REPCs were not targeted by Pax8-rtTA-mediated Cre recombination (Figure 1C). In contrast to the Pax8-rtTA model, $V h l$ inactivation in renal epithelium as well as in nonendothelial interstitial cells using a Cre transgene driven by the Pax3 promoter (4) increased renal Epo mRNA levels substantially (Supplemental Figure 1B). Taken together, our genetic data support the concept that renal epithelial cells do not have the capacity to synthesize EPO under conditions of constitutive HIF activation (Figure 1D).

In order to understand the polycythemia-independent effects of renal epithelial $V h l$ inactivation on the kidney, we established a Pax8-rtTA-based conditional Vhl knockout model, in which the supraphysiologic EPO production in the liver was ablated. For this, we generated $\mathrm{Vhl} / \mathrm{Epo}$ double-knockout mice (P8;Vhll/fl $\left.E p o^{f l / f l}\right)$, which were homozygous for both the Vhl-and the Epo-2-lox alleles. In this model, both alleles recombined with equal efficiency in kidney and liver (Figure 2A). Because REPCs were not targeted and because EPO synthesis in Vhl-deficient hepatocytes was ablated, we hypothesized that erythropoiesis in P8; $V h l^{f / f l}$

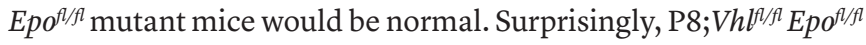
mutant mice developed significant anemia rapidly, within 2 weeks after doxycycline treatment was initiated (Hct: $28.8 \% \pm$ $0.8 \%$ vs. $43.4 \% \pm 0.6 \% ; \mathrm{Hb}: 8.7 \pm 0.4 \mathrm{~g} / \mathrm{dl}$ vs. $13.0 \pm 0.1 \mathrm{~g} / \mathrm{dl} ; \mathrm{rbc}$ : $6.2 \pm 0.2 \mathrm{M} / \mu \mathrm{l}$ vs. $9.2 \pm 0.1 \mathrm{M} / \mu \mathrm{l} ; n=5$ each) (Figure $2 \mathrm{~B}$ ). Reticulocyte counts and reticulocyte production index (RPI) were reduced, which indicated that anemia was hypoproliferative (Figure $2 \mathrm{C}$ ). In contrast, erythropoiesis in P8;Epo $o^{f / f l}$ single-knockout mice was not impaired, which is in line with our observation that REPCs were not targeted in the Pax8-rtTA-based genetic model and which established that anemia was the result of $V h l$ inactivation and not Epo inactivation in epithelial cells (Figure 2B). Renal Epo transcript levels were significantly reduced in P8;Vhll/fl Epo $o^{f / f l}$ mutant mice, indicating that $\mathrm{P} 8 ; V h l^{f / f l} E p o^{f / f l}$ mutants developed anemia due to insufficient renal Epo transcription, whereas nonanemic P8;Epo ${ }^{f l / f l}$ mutants produced normal renal Epo transcript levels at baseline (Figure 2D) and had normal sEPO responses to phlebotomy (Supplemental Figure 4B). Taken together, our findings demonstrate that acute ablation of $V h l$ in renal epithelial cells suppresses EPO production in the kidney and results in the rapid development of renal anemia.

Since anemia in patients with CKD develops when renal function declines by at least $50 \%$ and glomerular filtration rate (GFR) is less than $60 \mathrm{ml} / \mathrm{min}$ (3), we examined whether rapid

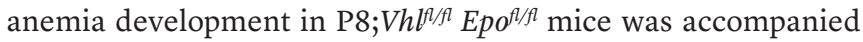
by decreased renal function. We measured blood urea nitrogen (BUN) levels and determined GFR by measuring FITC-inulin clearance. We furthermore evaluated renal blood flow (RBF) by ${ }^{99 m}$ Tc-MAG3 scintigraphy 2 weeks after completion of doxycycline treatment. We did not observe significant differences in either BUN levels (43.25 $\pm 1.8 \mathrm{mg} / \mathrm{dl}$ in P8; $V h l^{f l / f l} \mathrm{Epo}^{f l / f l}$ mutants vs. 36.75 $\pm 4.6 \mathrm{mg} / \mathrm{dl}$ in controls, $n=4 \mathrm{each})$, GFR values $(234.5 \pm 38.4 \mu \mathrm{l} /$ min in P8; Vhl ${ }^{f l / f l} \mathrm{Epo}^{f / f l}$ mutants vs. $218.0 \pm 13.0 \mu \mathrm{l} / \mathrm{min}$ in controls, $n=3$ each $)$, or $\operatorname{RBF}\left(0.36 \pm 0.01\right.$ relative activity/s in P8; Vhll/fl Epo $o^{f / f l}$ mice vs. $0.32 \pm 0.04$ relative activity/s in controls, $n=4$ each) between P8; Vhllf/fl $E p o^{f l / f l}$ and control mice (Supplemental Figure 2A). Plasma $\mathrm{Na}^{+}$and $\mathrm{K}^{+}$levels were normal, indicating that sodi- 
A

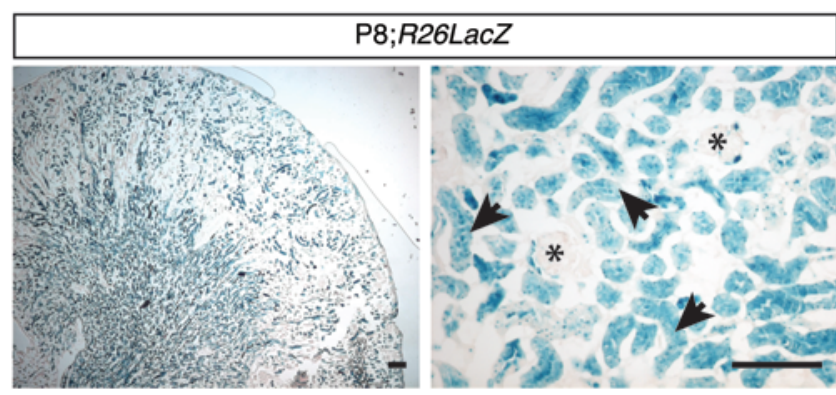

B

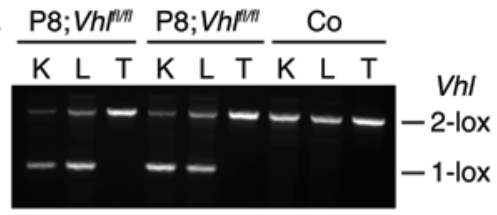

C

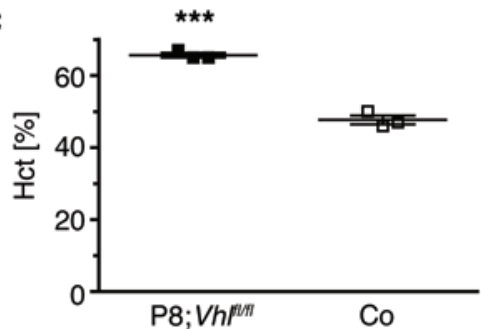

D
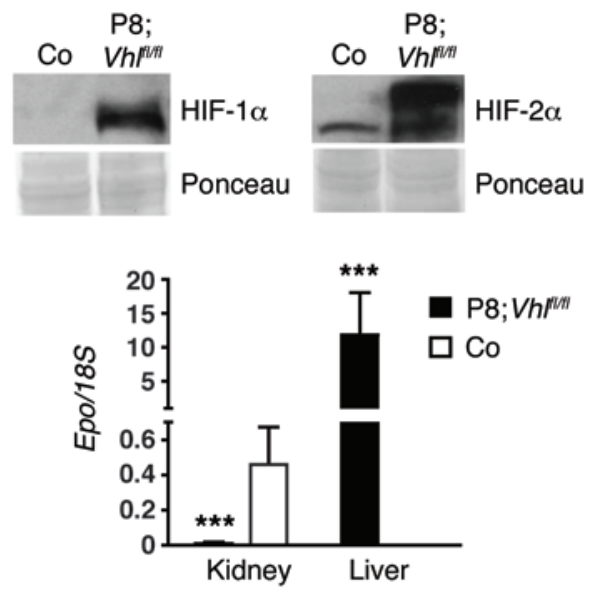

Co

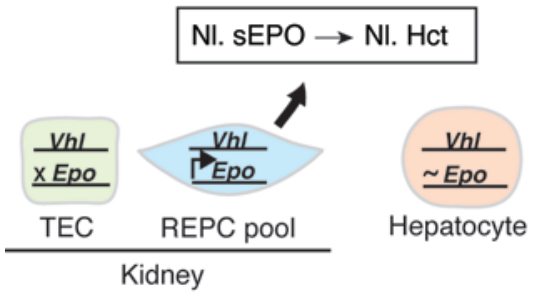

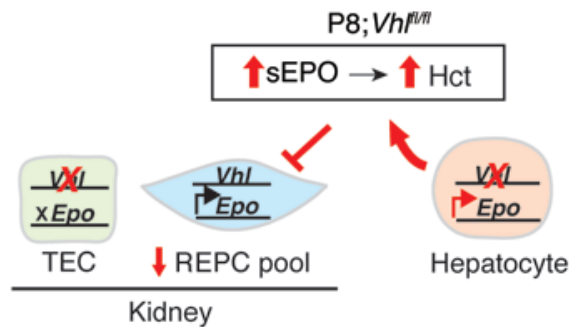

Figure 1. Pax8-rtTA-mediated inactivation of $\mathrm{Vhl}$ induces hepatic EPO and causes polycythemia. (A) Representative X-gal staining of the kidney from an R26R;Pax8-rtTA;LC-1 Cre-reporter mouse. Blue staining indicates cells that have undergone Pax8-rtTA-mediated Cre recombination. Cre activity was only detected in renal epithelial cells (arrows), whereas glomeruli were not stained (asterisks). (B) Left panel: PCR analysis of genomic DNA isolated from kidney $(\mathrm{K})$, liver (L), and tail (T) of P8; Vhffl/fl mutant and control (Co) mice 1 week after completion of doxycycline treatment. Right panel: HIF- $1 \alpha$ and HIF-2 $\alpha$ protein levels in whole-kidney lysates. Ponceau staining was used to demonstrate equal protein loading. (C) Hct values in individual P8; Vh/fl/fl mutant and control mice ( $n=3$ each) and relative Epo mRNA expression levels in kidneys and livers from $\mathrm{P} 8$; $V h f^{f / f f l}$ mutant and control mice ( $n=3$ each). (D) Schematic depicting the cellular contributions to SEPO under physiological conditions in control (left panel) and $\mathrm{P} 8 ; \mathrm{Vh} \mathrm{f}^{|l| f l}$ mice (right panel). The red $\mathrm{X}$ indicates Cre-mediated gene inactivation. The black $\mathrm{x}$ indicates that the Epo gene in tubular epithelial cells (TEC) is not transcribed. The tilde indicates that Epo is nondetectable in hepatocytes at baseline and does not contribute to plasma EPO levels under normoxic conditions. In P8; Vh/fl/fl mutants, EPO is produced in hepatocytes, while renal EPO output is physiologically suppressed. ${ }^{* *} P<0.001,2$-tailed Student's $t$ test. Shown are mean values \pm SEM. Scale bars: $200 \mu \mathrm{m}$. 2-lox, nonrecombined conditional allele; 1-lox, recombined allele. See Supplemental Figure 1 for additional information. um and water homeostasis was grossly intact (Supplemental Figure 2B). Furthermore, morphologic and molecular examination of P8; $V h l^{f l / f l} E p o^{f / f l}$ kidneys did not reveal any structural abnormalities, evidence of inflammation, or increased expression of renal injury markers, such as kidney injury molecule 1 (Kim1) and lipocalin-2 (Lcn2), compared with control (Supplemental Figure 2C). Thus, our data indicate that the rapid development of renal anemia in P8; Vhll/fll Epo ${ }^{f / f l}$ mice did not result from a loss of renal function or structural changes in the kidney and was not associated with renal inflammation.

Inactivation of epithelial Vhl reduces the number of REPCs. Previous studies have shown that the number of REPCs, which is dependent on the degree of renal hypoxia, controls renal EPO production (for a detailed overview see ref. 3). To examine whether acute $V h l$ ablation in renal epithelium affected the number of REPCs, we used high-resolution in situ hybridization to visualize REPCs in nonphlebotomized and phlebotomized P8; Vhllf/ll Epo ${ }^{f / f l}$ and Cre control mice. We found that the number of REPCs at baseline was significantly reduced in $\mathrm{P} 8 ; V h l^{f^{l / f l}} E \mathrm{Ep} 0^{f / / l}$ mice compared with Cre control animals $\left(1.7 \pm 0.8\right.$ cells $/ \mathrm{mm}^{2}$ in mutants and $9.8 \pm 0.5$ cells/ $\mathrm{mm}^{2}$ in control mice). To examine EPO production in $\mathrm{P} 8$; Vh/t/fl $E p o^{f l f l}$ kidneys in response to anemia, we induced similar degrees of anemia in P8; Vhl $l^{f / f l} E p o^{f / f l}$ and control mice by phelobotomy (Hct: $14.4 \% \pm 0.6 \%$ and $16.7 \% \pm 1.4 \%$, respectively). We found that the predicted increase of REPC number was severely blunted in P8; Vhllf/fl Epo $o^{f / f l}$ mice $\left(37.1 \pm 12.4\right.$ cells $\left./ \mathrm{mm}^{2}\right)$ compared with control mice $\left(210.2 \pm 19.1\right.$ cells $/ \mathrm{mm}^{2}$ ) (Figure $3 \mathrm{~A}$ ). The decrease in REPC number was associated with a significant reduction in sEPO levels as well as a reduction in renal Epo transcript levels (Supplemental Figure 3A). In contrast with P8;Vhll/fl Epo fl/fl mutants, the level of renal Epo induction was similar between phlebotomized P8;Epo $o^{f / f l}$ and control mice, demonstrating that Pax8-rtTA-mediated inactivation of Epo alone in renal epithelium did not affect the physiologic behavior of renal interstitial cells with EPO-producing potential (Supplemental Figure 3B).

Renal cortical $\mathrm{PtO}_{2}$ is elevated in $\mathrm{P} 8 ; \mathrm{Vh}^{f^{l / f l}} \mathrm{Epo}^{f l / f l}$ mice. Because the size of the REPC pool is $\mathrm{PtO}_{2}$ dependent, we next asked whether the reduction in REPC number in P8; Vhll/fl $E p o^{f l / f l}$ mutants was associated with changes in renal $\mathrm{PtO}_{2}$. Cortical $\mathrm{PtO}_{2}$ was determined with a Clark electrode in 3 groups of mice: P8;Vhll/fl Epo $o^{f / f l}$ mutants (Hct: $33.0 \% \pm 0.8 \%$, obtained in 6 of 10 experimental mice), normemic control mice (Hct: $44.8 \% \pm 1.1 \%$, obtained in 6 of 10 experimental mice), and phlebotomized anemic control mice (Hct: $20.1 \% \pm 1.5 \%, n=7$ ) (Supplemental Figure 3C). Despite 


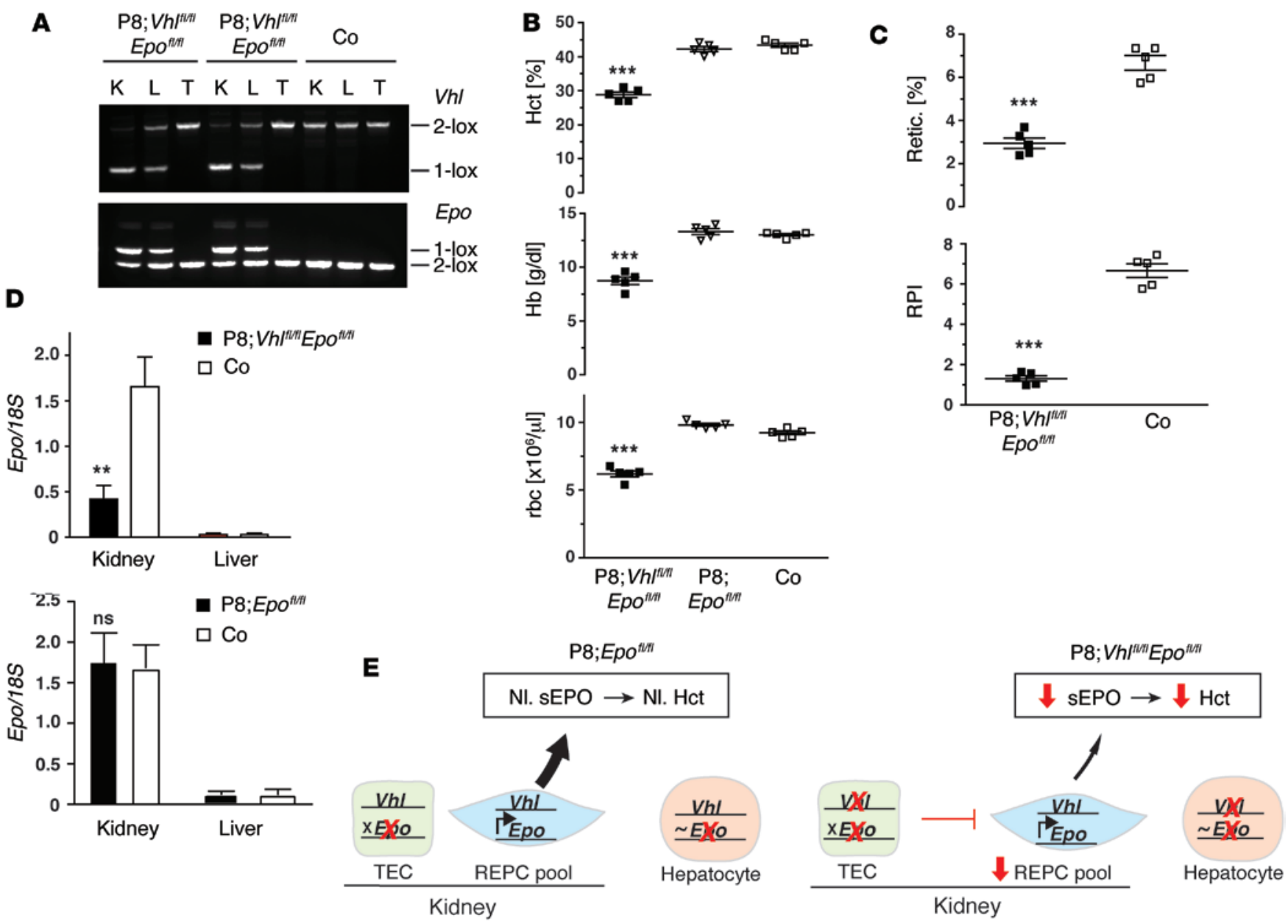

Figure 2. Renal epithelial Vhl inactivation results in anemia. Hematologic effects of Pax8-rtTA-mediated inactivation of Vhl in the absence of hepatic EPO induction. (A) Genomic PCR analysis of DNA isolated from kidney, liver, and tail of P8;Vh/fl/fl Epo fl/fl and Cre- control mice. (B) Hct, Hb, and rbc counts in P8;Vh/fl/fl $E p 0^{f / f f l}, \mathrm{P} 8 ; E p 0^{f / f f l}$, and control ( $n=5$ each). (C) Corresponding reticulocyte counts (Retic.) and RPI in P8;Vh/fl/fl $E p 0^{f / f f}$ and control mice ( $n=5$ each). (D) Relative renal and hepatic Epo mRNA levels in P8; Vh/fl/fl Epofl/fl and control mice (top panel, $n=5$ each) and P8;Epo fl/fl (bottom panel; $n=6$ kidney samples and $n=3$ liver samples) in comparison with control mice ( $n=8$ kidney samples and $n=3$ liver samples). (E) Schematic depicting cellular contributions to sEPO levels in

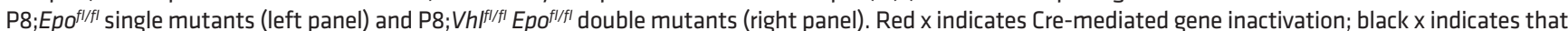
the Epo gene is not transcribed in tubular epithelial cells. The tildes indicate that Epo is not detectable in hepatocytes under baseline conditions. EPO production in the liver is no longer increased in P8;Vh/fl/fl Epo fl/fl double mutants (right panel). ${ }^{*} P<0.01$; ${ }^{* *} P<0.001$, 1-way ANOVA followed by Dunnett's post hoc analysis (B); unpaired 2-tailed Student's $t$ test (C and D). Shown are mean values \pm SEM. 2-lox, nonrecombined conditional Vhl or Epo allele; 1-lox, recombined allele. See Supplemental Figure 2 for additional information.

the presence of anemia, cortical $\mathrm{PtO}_{2}$ in $\mathrm{P} 8 ; \mathrm{Vh}^{f / f l} \mathrm{Epo^{f/f }}$ mice was significantly elevated compared with that in normemic controls $(39.26 \pm 2.2 \mathrm{mmHg}$ vs. $30.8 \pm 1.3 \mathrm{mmHg} ; n=10$ for both groups; $P<0.01)$. $\mathrm{PtO}_{2}$ values in anemic control mice averaged $14.2 \pm 2.6$ $\mathrm{mmHg}$, which was expected, as oxygen-carrying capacity in these mice was significantly reduced $(n=7)$ (Figure 3B). Arterial $\mathrm{O}_{2}$ saturation $\left(\mathrm{SaO}_{2}\right)$ or $\mathrm{PaO}_{2}$ was found to be indistinguishable between mutants and control mice, indicating that differences in arterial oxygenation did not account for the observed differences in cortical $\mathrm{PtO}_{2}$ (Supplemental Figure 3C).

To gain additional insights into the regulation of EPO in P8; Vh/ ${ }^{R / f l} E p 0^{f / f l}$ mutants, we asked whether EPO suppression in $\mathrm{P} 8 ; V h l^{f / f l} E p o^{f / f l}$ mutants was exclusively dependent on $\mathrm{PtO}_{2}$. Inhibition of PHD catalytic activity stabilizes HIF- $2 \alpha$ and increases EPO synthesis irrespective of $\mathrm{PtO}_{2}$. Assuming equal bioavailability, we predicted that systemic administration of a potent pan-HIF prolyl-4-hydroxylase inhibitor (PHI) would result in equally efficient HIF activation in kidneys from mutant and control mice and lead to comparable Epo transcription if the PHD/HIF/EPO axis was functionally intact in interstitial fibroblast-like cells. For the pharmacologic inhibition of HIF prolyl-4-hydroxylation, we utilized tool compound GSK1002083A (GlaxoSmithKline). We have previously shown that GSK1002083A resulted in robust activation of the HIF/EPO axis regardless of $\mathrm{PtO}_{2}$ levels (4). While PHI treatment led to an approximately 16-fold increase in renal Epo mRNA

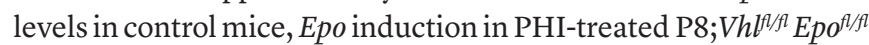
mutants was blunted $(\sim 7$-fold increase compared with control; $n=8$ each; $P<0.001$ ) (Figure $3 C$ ). This finding suggested that the PHD/ HIF/EPO axis in P8; Vhllf/l $\mathrm{Epo}^{f / f l}$ mutants was less sensitive to PHD inhibition and raised the possibility that anemia development in these mutants was not exclusively due to increased cortical $\mathrm{PtO}_{2}$ and may also have involved $\mathrm{PtO}_{2}$-independent mechanisms. 

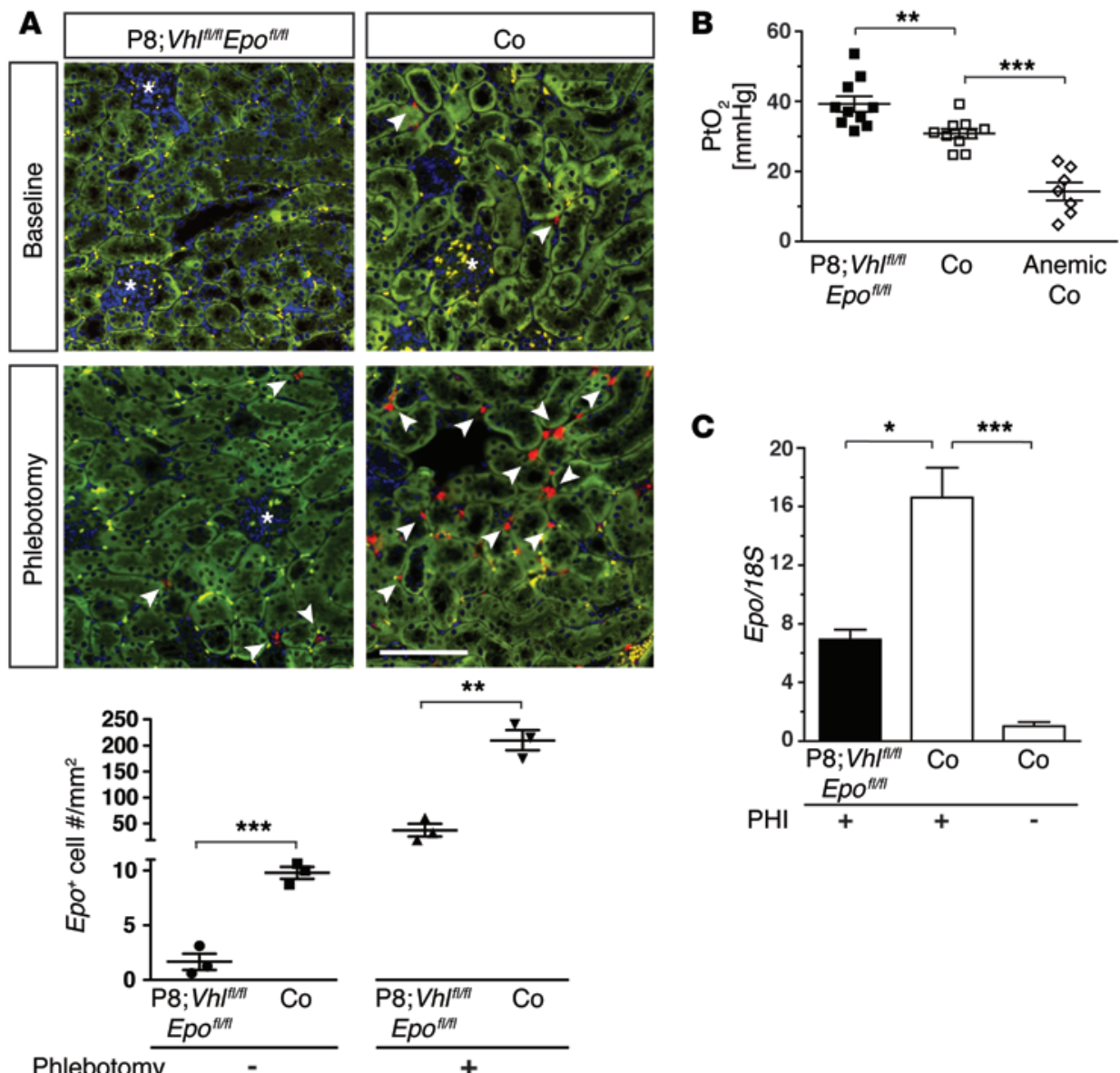

C

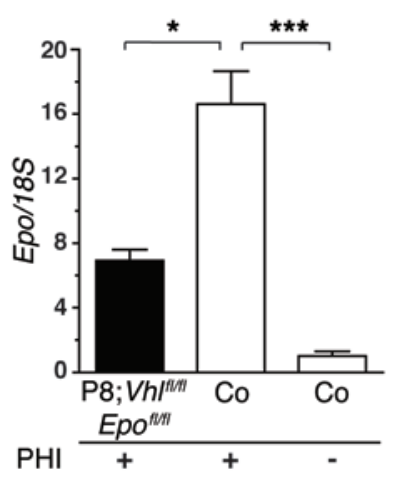

Figure 3. The number of EPO-producing cells is reduced in P8; Vhf ${ }^{f / f l} \mathrm{Epo}^{f / f l}$ mutants. (A) Representative images of Epo mRNA detection by RNA in situ hybridization (red spots) in kidney section from P8; Vh/fl/fl Epo ${ }^{f / / f l}$ and Cre-control mice at baseline and following phlebotomy. DAPI (blue) was used for nuclear staining. Number of Epo-expressing cells was counted in each renal cross-section and expressed as number of cells $/ \mathrm{mm}^{2}$ ( $n=3$ each). (B) Shown are the results from cortical tissue $\mathrm{O}_{2}$ partial pressure $\left(\mathrm{PtO}_{2}\right)$ measurements in $\mathrm{P} 8 ;\left.\mathrm{Vh}\right|^{\mathrm{fl|fl} \mid}$ $E p o^{f l / f l}$ and control mice. Each data point represents the average mean value of at least 3 measurements per individual mouse ( $n=10,10$, and 7 , respectively). (C) Relative Epo mRNA levels in wholekidney homogenates from P8; Vh/fl/fl

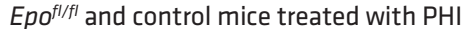
CSK1002083A 1 week after completion of doxycycline treatment $(n=8,8$, and 3 , respectively). ${ }^{*} P<0.05 ;{ }^{* *} P<0.01$; ${ }^{* * *} P<0.001$, unpaired 2-tailed Student's $t$ test (A); 1-way ANOVA followed by Dunnett's post-hoc analysis (B and C). Shown are mean values \pm SEM. Scale bar: $100 \mu \mathrm{m}$. See Supplemental Figure 3 for additional information.
Vhl ablation in renal epithelium shifts renal metabolism toward glycolysis. We next asked whether changes in renal metabolism could be responsible for the increased cortical $\mathrm{PtO}_{2}$, as HIF regulates cellular energy metabolism by increasing glucose utilization and glycolysis and shifts oxidative phosphorylation toward glycolysis (17) by blocking the conversion of pyruvate to acetyl-CoA via inhibition of pyruvate dehydrogenase (PDH) $(18,19)$. To assess the effects of pan-tubular HIF activation on renal energy metabolism in vivo, we first performed microarraybased genome-wide mRNA expression analysis in whole-kidney homogenates from $V h l$-deficient mice. We found that a substantial number of genes involved in glucose and fatty acid metabolism were differentially expressed $(>1.5$-fold change with an adjusted $P$ value of $<0.05)$. The mRNA levels of most genes regulating glycolysis and glucose metabolism were increased (Figure 4). These included hexokinase 2 (Hk2), glucosephosphate isomerase 1 (Gpi1), aldolase C (Aldoc), triosephosphate isomerase 1 (Tpi1), phosphogylcerate kinase 1 (Pgk1), phosphoglycerate mutase 1 (Pgam1), pyruvate kinase M2 (Pkm2), and lactate dehydrogenase A (Ldha) (Figure 4A). Gene-expression changes were in support of our hypothesis that renal epithelial energy metabolism in Vhl-deficient kidneys had shifted from oxidative phosphorylation toward glycolysis. Consistent with a reduction in oxidative phosphorylation was the differential expression of genes involved in fatty acid storage and lipogenesis, which suggested a dysregulation of fatty acid metabolism (Figure 4B). In addition, we observed decreased expression of mRNAs encoding multiple ATP-consuming renal transporters, such as the sodium-dependent dicarboxylate transporter (Nadc1/Slc13a2), the sodium/sulfate symporter (NaS2/Slc13a4), sodium/hydrogen exchanger 2 (Nhe2/Slc9a2), the ammonium transporter rhesus blood groupassociated B glycoprotein ( $\mathrm{hhbg} / \mathrm{Slc42a2}$ ), the sodium-dependent neutral amino acid transporter (B[0]at1/Slc6a19), sodiumdependent phosphate transport protein 2B (Npt2b/Slc34a2), and the organic anion transporter 2 (Oat2/Slc22a7), raising the possibility that epithelial $V h l$ deletion led to a reduction in renal energy demand (Figure 4C). Microarray data were validated by realtime PCR analysis (Figure 4D). The metabolic gene-expression changes were furthermore reflected in increased glucose uptake in the renal medulla, as assessed by ${ }^{14} \mathrm{C}$-deoxy-D-glucose (2-DG) uptake measurements, and in elevated total kidney renal pyruvate and lactate levels, as measured by mass spectrometry in P8; Vhlllfll $E p o^{f l / f l}$ mice (Figure 4, E and F). Taken together, our data suggest that $V h l$ inactivation in renal epithelium led to marked changes in kidney metabolism and shifted renal energy metabolism from oxidative metabolism toward glycolysis, which in turn predicted a decrease in renal $\mathrm{O}_{2}$ consumption.

Vhl ablation in renal epithelium decreases $\mathrm{O}_{2}$ consumption. To assess renal $\mathrm{O}_{2}$ consumption in $V h l$-deficient kidneys, we performed high-resolution respirometry in mechanically permeabilized cortical and medullary tissue samples from P8;Vhllfll and P8; Vhlll/fl $E p o^{f l / f l}$ mutant kidneys. We assessed respiratory activity 
A

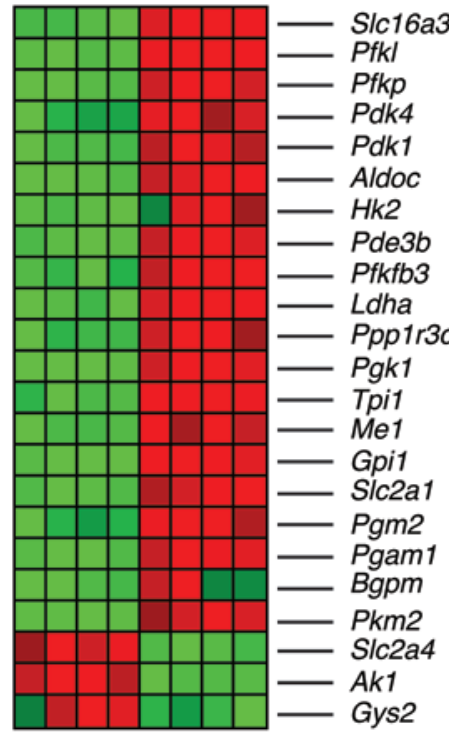

B

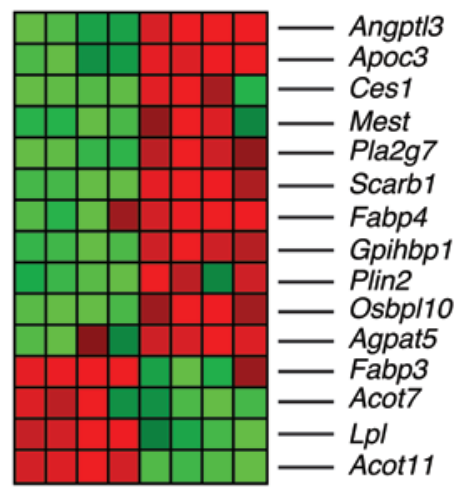

C

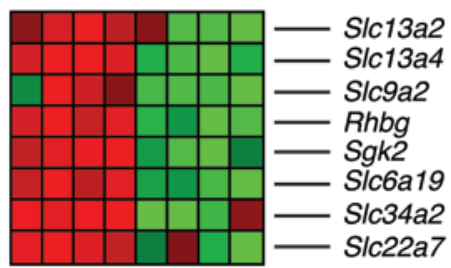

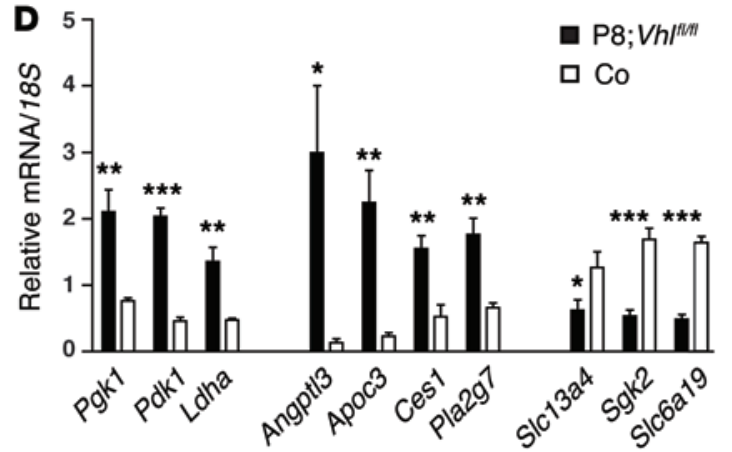

F

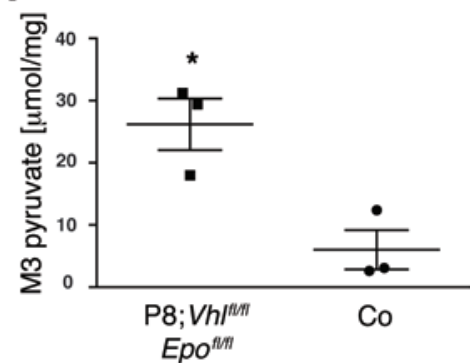

E

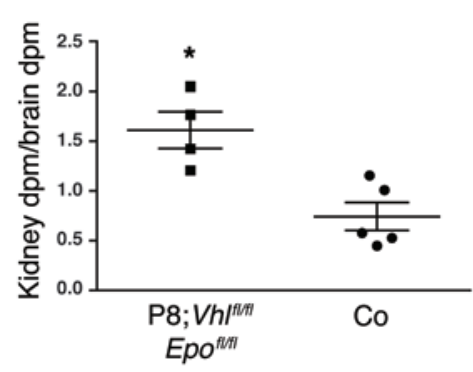

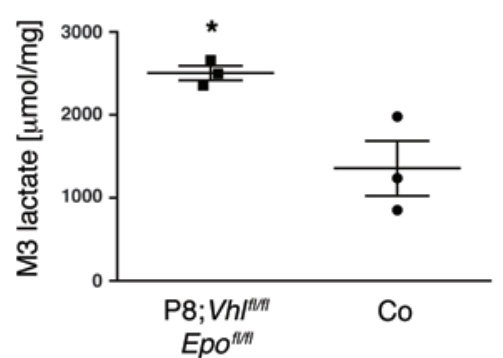

Figure 4. Epithelial Vhl ablation alters renal metabolism. Differential regulation of genes involved in glycolysis (A), fatty acid metabolism (B), and renal transport function (C) by genome-scale RNA expression profiling in kidneys from P8; Vh/flft and Cre- control mice ( $n=4$ each). Shown are differentially regulated metabolic genes that were either significantly up- or downregulated ( $\geq 1.5$-fold). Color intensity is scaled within each row so that the highest expression value corresponds to bright red and the lowest to bright green. Each column represents expression levels in an individual kidney. (D) Verification of gene-expression analysis by quantitative PCR for key target genes from P8; Vh/f/ffl and Cre control mice ( $n=6$ each). (E) Assessment of renal glucose metabolism by quantification of 2-DC uptake in renal medulla from P8;Vh/f/ffl $E p 0^{f / f / f}$ and control mice $\left(n=3\right.$ each). (F) Renal M3 pyruvate and M3 lactate concentrations in total kidney extracts from P8;Vh/f/ffl Epo $0^{f / f / l}$ and control mice $\left(n=3\right.$ each). ${ }^{*} P<0.05 ;{ }^{* *} P<0.01 ;{ }^{* *} P<0.001$, unpaired 2 -tailed Student's $t$ test. Shown are mean values \pm SEM.

at complex I (cI), complex II (cII), and complex IV (cIV), utilizing complex-specific substrates and inhibitors. In cortical and medullary tissue preparations, state 2 respiration (lack of ADP in the presence of substrate) and the activities of cI, cII, and cIV were mark-

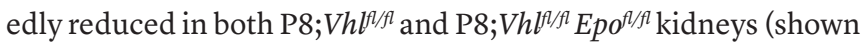
here are data for P8; $V h l^{l / f l} E p o^{f / f l}$ and control kidneys; $n=5$, respectively) (Figure 5A). Because HIF regulates mitochondrial biogenesis (20), we next assessed mitochondrial mass in Vhl-deficient mice. For this, we measured the activity of mitochondrial citrate synthase (CS) in cortical and medullary tissue samples (21). Both cortical and medullary CS activity, which typically correlate with mitochondrial mass, were significantly reduced, by approximately $30 \%$ and $50 \%$, respectively. This finding was furthermore reflected in a comparable reduction of mitochondrial DNA (mtDNA)
(Figure 5, B and C). Single mitochondrial respiration in cortex, which was assessed by normalizing $\mathrm{O}_{2}$ consumption to CS activity, was not different between P8;Vht $t^{\ell / f l} E p o^{f / f l}$ and control mice $\left(n=5\right.$ each). Normalized $\mathrm{O}_{2}$ consumption in medullary preparations was also unchanged for state 2 , cI, and cII respiration, while single mitochondrial $\mathrm{O}_{2}$ consumption through cIV was increased (Figure 5D). In summary, our results demonstrate that reduced renal $\mathrm{O}_{2}$ consumption in $V h l$-deficient mice was associated with a reduction in mitochondrial mass.

Cocontribution of epithelial HIF-1 $\alpha$ and HIF-2 $\alpha$ to anemia devel-

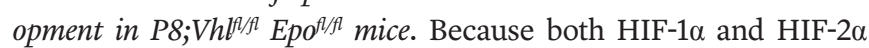
were detected in renal tissue from $V h l$-deficient mice (Figure 1), we

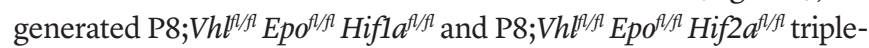
knockout mice to characterize the individual contributions of HIF-1 $\alpha$ 
A
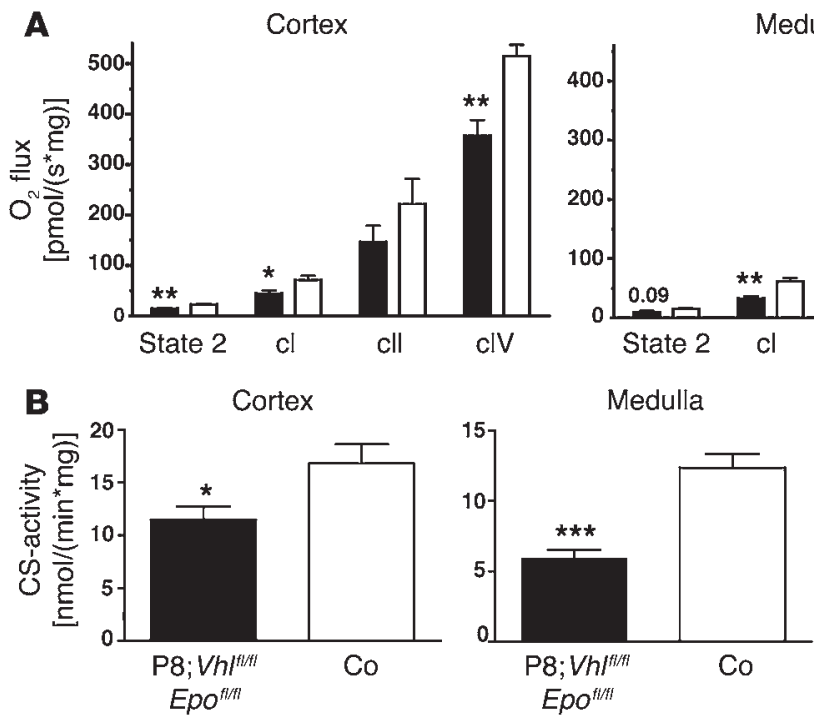

Medulla
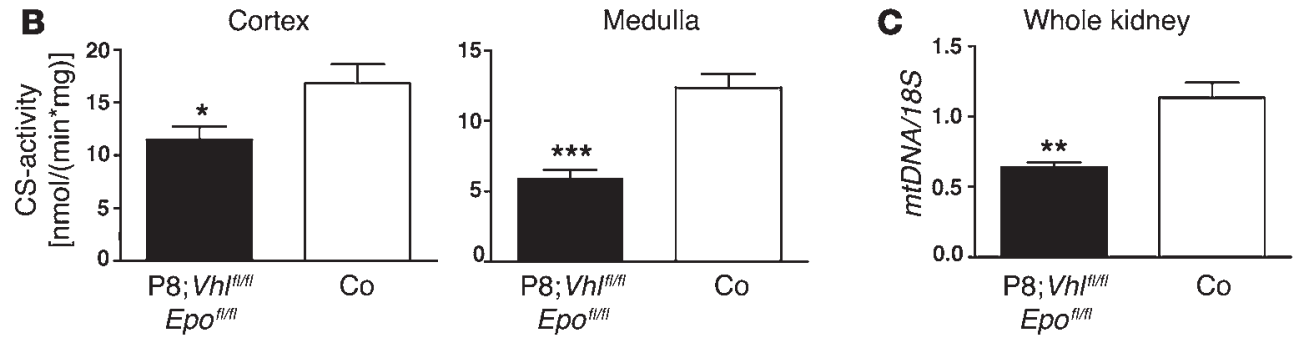

D
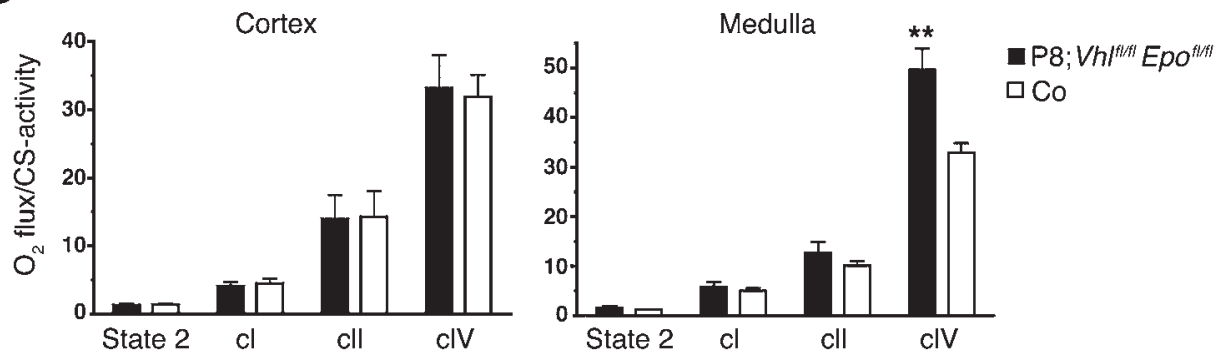

Figure 5. Renal epithelial Vhl ablation reduces respiratory capacity and mitochondrial mass. (A) $\mathrm{O}_{2}$ flux measurements in mechanically permeabilized cortical and medullary tissue samples from $\mathrm{P} 8 ; \mathrm{Vh}^{|f| f \mid f l} \mathrm{Epo}^{\mathrm{fl} / f l}$ and $\mathrm{Cre}^{-}$control mice ( $n=5$ each). (B) CS activity of cortical and medullary tissue samples from $\mathrm{P} 8 ; \mathrm{Vh}^{\mid f / / f l} \mathrm{EpO}^{f / f l}$ and control mice ( $n=5$ each). (C) Relative quantification of mitochondrial mass by real-time PCR analysis of whole-kidney genomic DNA samples from $\mathrm{P} 8 ; \mathrm{Vh}^{|f| / f l} E p \mathrm{O}^{f l / f l}$ and control mice ( $n=5$ each). Relative mtDNA levels were normalized to nuclear encoded 185 DNA. (D) $\mathrm{O}_{2}$ fluxes normalized to CS activity ( $n=5$ each). ${ }^{*} P<0.05$; ${ }^{* *} P<0.01$; ${ }^{* * *} P<0.001$, unpaired 2-tailed Student's $t$ test. Shown are mean values \pm SEM. State 2 , basal respiration in the presence of substrate but lack of ADP. and HIF-2 $\alpha$ to the development of anemia and metabolic reprogramming in P8;Vhtl/fl Epo $o^{f / f l}$ double mutants. Genomic PCR analysis was used to confirm that efficient recombination of all conditional alleles $\left(V h l^{l / f l}, E p o^{f / f l}\right.$, and Hifl $a^{t / f l}$ or Hif $\left.2 a^{f / f l}\right)$ was achieved, and only mice with efficient recombination of the respective alleles in renal tissue were included in the analysis (Supplemental Figure 4A). Complete blood count analysis showed that erythropoiesis remained impaired

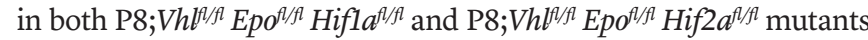
(Hct: $30.0 \% \pm 1.3 \%$; Hb: $9.2 \pm 0.3 \mathrm{~g} / \mathrm{dl} ; \mathrm{rbc}: 6.4 \pm 0.2 \mathrm{M} / \mu \mathrm{l} ; n=6$ and Hct: $34.3 \% \pm 0.9 \%$; Hb: $10.7 \pm 0.2 \mathrm{~g} / \mathrm{dl} ; \mathrm{rbc}: 7.9 \pm 0.2 \mathrm{M} / \mu \mathrm{l} ; n=3)$ (Figure 6A), suggesting that both HIF- $\alpha$ homologs contributed to the development of anemia in P8; Vh/t/fl Epo $o^{f / f l}$ mice.

Although renal Epo transcript levels in P8;Vhll/fll Epo ${ }^{f / / f l} H i f 1 a^{f l / f l}$

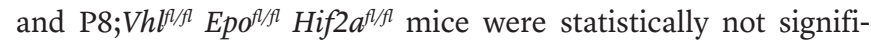
cantly different from those of control animals (Figure 6A), they were inadequate for the degree of anemia found in triple-mutant mice. Anemia of nonrenal etiology is usually characterized by an inverse linear relationship between Hct and the decadic logarithm of sEPO concentration. This relationship is altered in anemia associated with CKD, as the diseased kidney fails to respond to hypoxic stimuli with adequate EPO production (22). sEPO levels

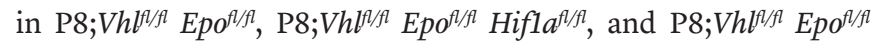
Hif $2 a^{f l / f l}$ mutant mice were inappropriately low for the degree of anemia that was present, whereas a linear decrease in Hct was associated with the expected logarithmic increase in sEPO levels in both $\mathrm{P} 8 ; E p o^{f / f l}$ and control mice (Supplemental Figure 4B).

To investigate whether HIF-1 and HIF-2 served redundant functions with regard to the observed changes in metabolic gene expression in P8;Vhllt/ll$E p o^{t / f l}$ mice, we measured expression levels of genes involved in glycolysis ( $P g k 1$ and pyruvate dehydrogenase kinase $1[P d k 1]$ ), lipid metabolism (angiopoietin like 3 [Angptl3] and apolipoprotein C-III [Apoc3]), and transport functions (Slc13a4 and serum/glucocorticoid regulated kinase 2 [Sgk2]). As shown in Figure 6B, upregulation of glycolytic gene expression appeared to be exclusively HIF-1 dependent, whereas the expression of Angptl3 and Apoc3 appeared to be HIF-2 dependent. While we could not attribute the suppression of transport genes Slc13a4 and $S g k 2$ to a specific HIF- $\alpha$ homolog, coinactivation of either Hifla or Hif2a in P8; Vhllf/fl Epo ${ }^{f / f l}$ mice was sufficient to increase mitochondrial mass (Figure 6C). Taken together, our genetic data suggest that the development of anemia in P8;Vhllf/ll Epo $o^{f / f l}$ mice required both HIF-1 and HIF-2 activation and could not be attributed to one HIF homolog alone.

Regulation of EPO synthesis by tubular epithelial cells is HIF dependent and localizes to the proximal nephron. Because we dissected the role of HIF-1 and HIF-2 in the development of anemia in Vhl-deficient mice and found that inactivation of either homolog did not restore normal erythropoiesis, we investigated to what degree anemia development in P8;Vhll/fl Epo $o^{f / f l}$ mice required intact signaling through both HIF- $\alpha$ homologs. For this, we bred mice that carried a floxed Hiflb (Arnt) allele to P8; Vhll/fl $E p o^{f / f l}$ mice, generating P8;Vhll/fl $E p o^{f / f l} A r n t^{f / f l}$ triple-knockout mice. ARNT serves as the $\beta$-subunit for both HIF-1 and HIF-2 heterodimers, and its inactivation abolishes canonical HIF signaling in renal epithelium (23). Concomitant ablation of Arnt restored hematologic parameters to normal values, as no significant differ- 

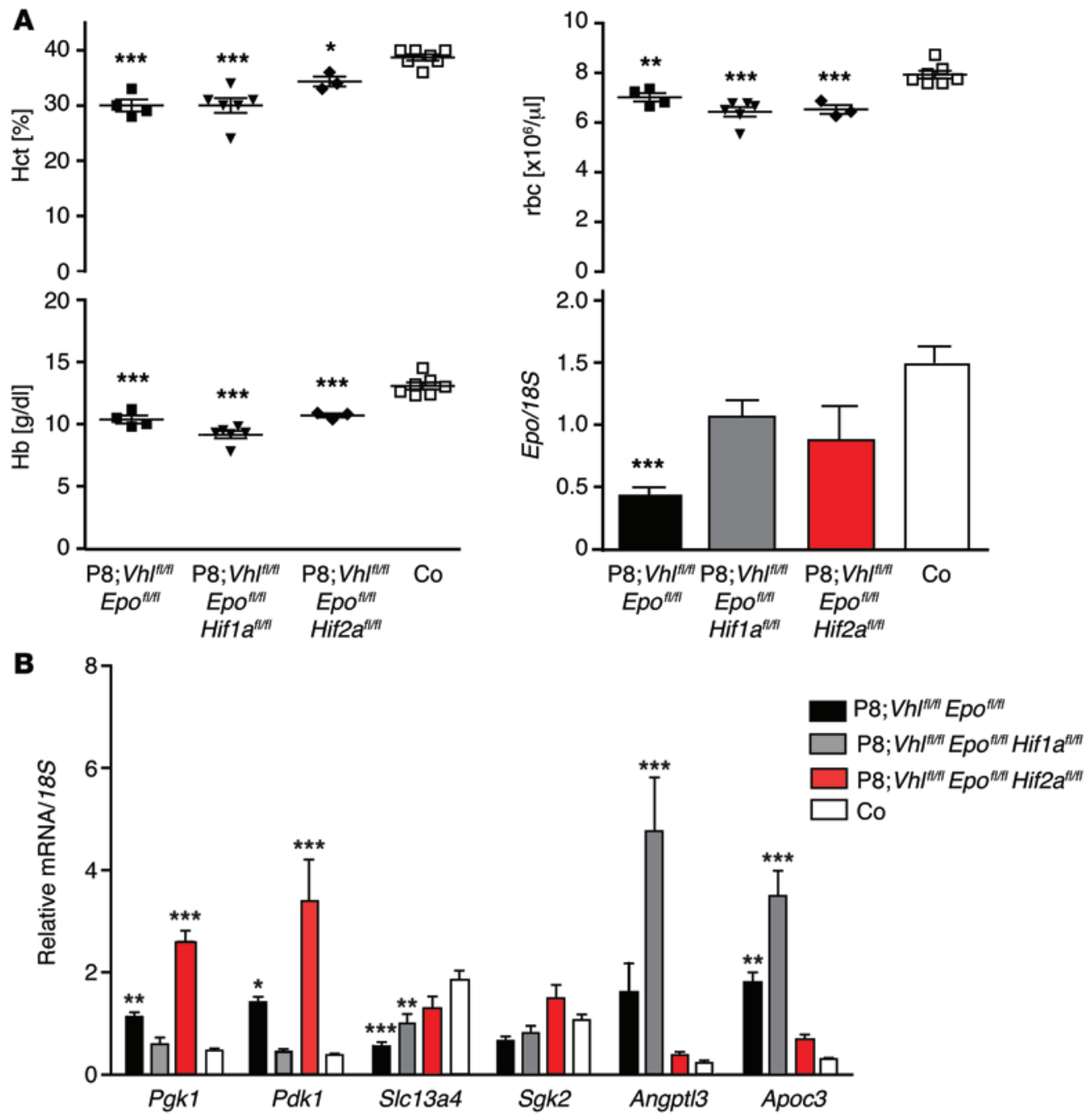

Figure 6. Epithelial HIF-1 and HIF2 coregulate renal Epo production and metabolism. (A) Shown are $\mathrm{Hct}, \mathrm{Hb}$, and rbc counts as well as renal Epo mRNA levels in P8; Vh/fl/fl

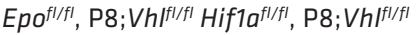
Hif $2 a^{f l / f f}$, and $\mathrm{Cre}^{-}$control mice ( $n=4,6,3$, and 7, respectively). (B) Renal mRNA expression of metabolic genes involved in glucose metabolism, fatty acid metabolism, and renal transport in P8;Vh/fl/fl Epo ${ }^{f l / f l}, \mathrm{P} 8 ;\left.\mathrm{Vh}\right|^{\mid f / f l}$ Hif1 $a^{f / f l}, \mathrm{P} 8 ; V^{\prime \mid f l / f l} H i f 2 a^{f l / f l}$, and Cre- control mice ( $n=4,6,3$, and 7 , respectively). (C) Relative quantification of mitochondrial mass by real-time PCR analysis of wholekidney genomic DNA samples from $\mathrm{P} 8 ; \mathrm{Vh}^{f / / f l} E p 0^{f / / f l}, \mathrm{P} 8 ; \mathrm{Vh} h^{f / / f l}$ Hif1 $a^{f / f l}, \mathrm{P} 8 ; \mathrm{Vh}^{|f / f|} \mathrm{Hif2} a^{f l / f l}$, and Cre- control mice $(n=4,6,3$, and 7, respectively). ${ }^{*} P<0.05 ;{ }^{*} P<$ $0.01{ }^{* * *} P<0.001$, 1-way ANOVA followed by Dunnett's post hoc analysis ( $\mathbf{A}$ and $\mathbf{C}$ ); unpaired 2-tailed Student's $t$ test (B).

Shown are arithmetic mean values \pm SEM. See Supplemental Figure 4 for additional information.

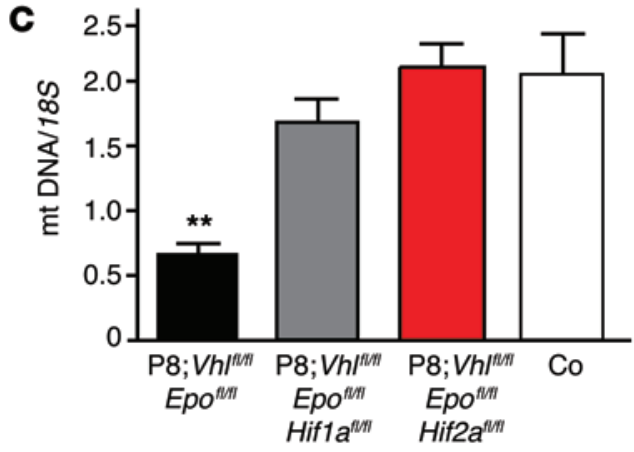

ences in $\mathrm{Hct}, \mathrm{Hb}$, or rbc were found between P8; Vhll/fll $\mathrm{Epo}^{f l / f l}$ Arnt $t^{f / / f l}$ and control mice (Figure 7A). This finding is consistent with renal

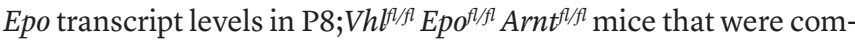
parable to those of control mice (Figure 7A). These genetic data establish that the suppression of renal EPO production in P8; $V h l^{f / f l}$ $E p o^{f / f l}$ mice required intact HIF signaling.

To investigate which specific segment of the nephron is involved in the suppression of renal EPO production, we examined several conditional $V h l$ knockout lines in which different nephron segments were targeted (Pepck-Cre, renal proximal tubule; ThpCre, medullary thick ascending limb of Henle and early distal tubule; Hoxb7-Cre, collecting duct). Since Pepck-Cre-driven conditional ablation of $\mathrm{Vhl}$ leads to polycythemia due to induction of hepatic EPO production (23), we generated Pepck-Cre Vhll/fl Epo $o^{f / f l}$ double-knockout mice. Similar to the P8; Vhlt/fll Epo ${ }^{f / f l}$ mutants, Pepck-Cre Vhll/fl Epo $o^{f / f l}$ double-knockout mice were anemic (6 to 8 weeks old, Hct: $29.4 \% \pm 1.5 \%$; Hb: $6.8 \pm 0.4$ g/dl; rbc: $7.1 \pm 0.3 \times$ $\left.10^{6} / \mu \mathrm{l} ; n=6\right)$, whereas mice with heterozygous deletion of $\mathrm{Vhl}$ and homozygous deletion of Epo (Pepck-Cre $\mathrm{Vh}^{\mathrm{fl} / \mathrm{+}} \mathrm{Ep} \mathrm{o}^{f / f l}$ ) displayed normal erythropoiesis (Hct: $39.0 \% \pm 0.7 \%$; Hb: $12.9 \pm 0.2 \mathrm{~g} / \mathrm{dl}$; rbc: $8.8 \pm 0.1 \times 10^{6} / \mu \mathrm{l} ; n=4$ ), (Figure 7B). Hematological analysis of 6- to 8-week-old Thp-Cre Vhlt/fl $(n=5)$ and Hoxb7-Cre Vht/f/l 

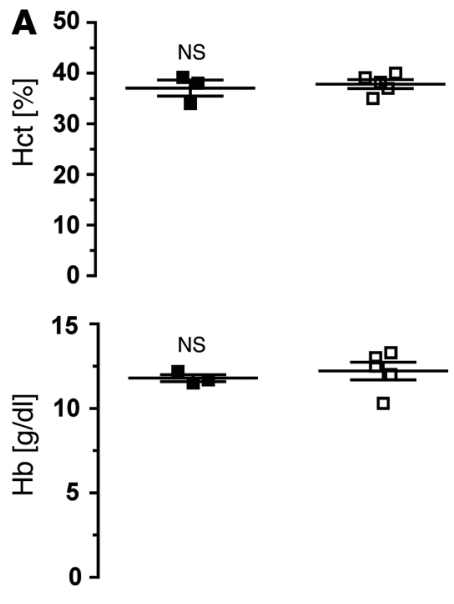

B
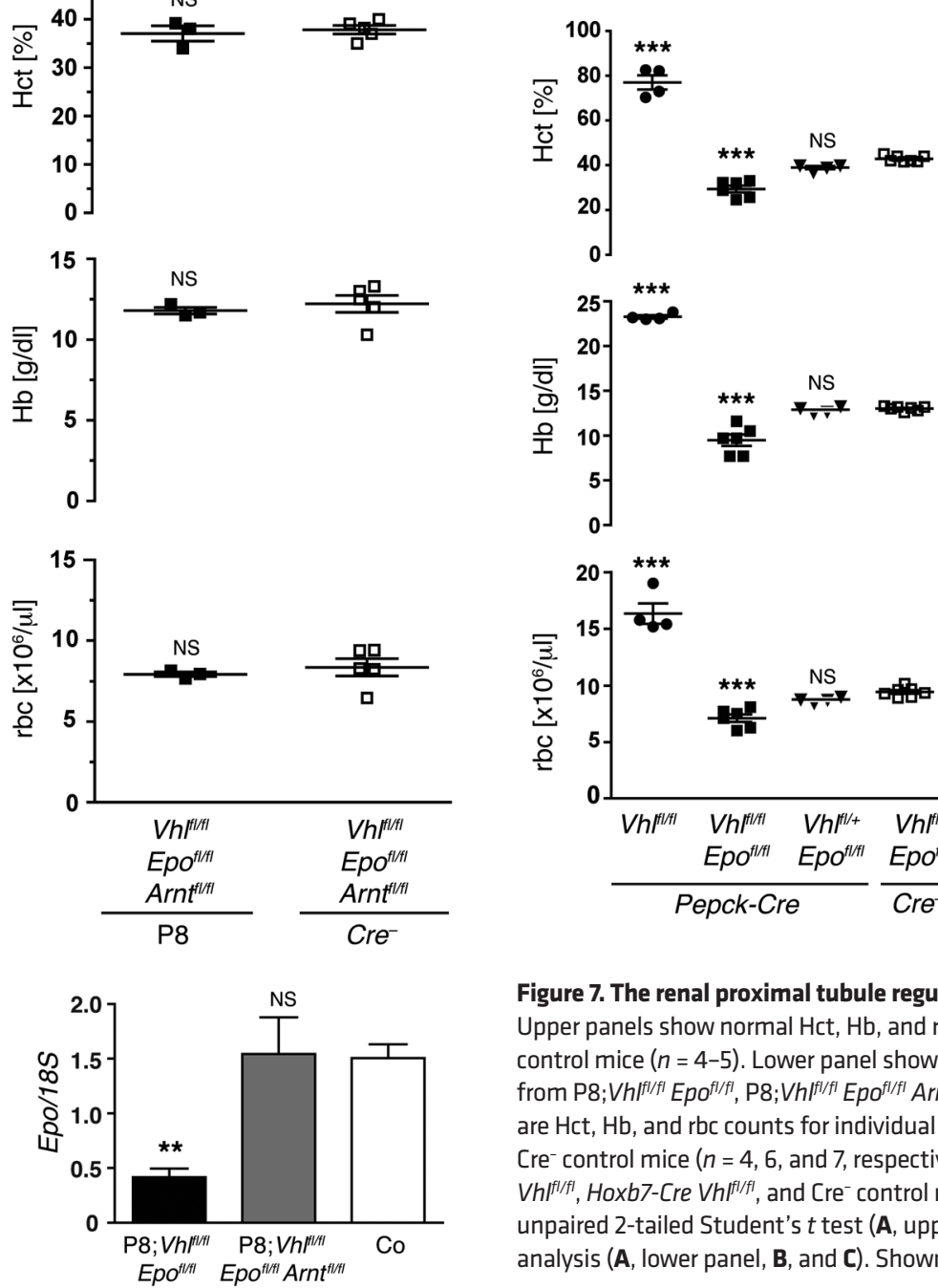
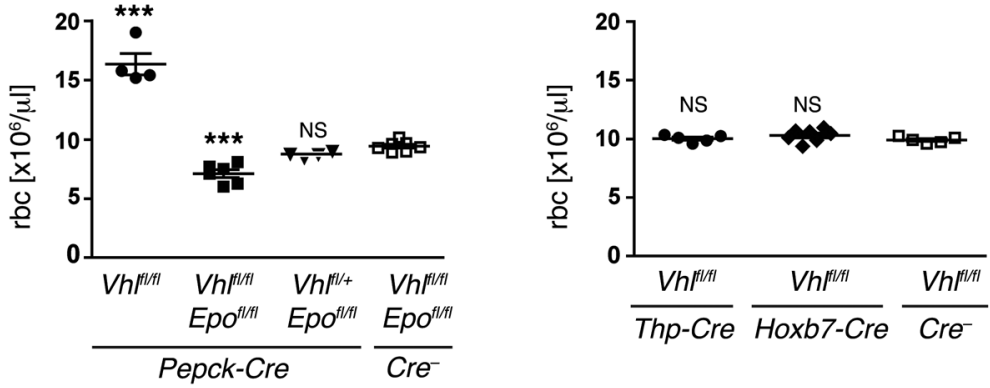

Figure 7. The renal proximal tubule regulates EPO production in an HIF-dependent manner. (A) Upper panels show normal $\mathrm{Hct}, \mathrm{Hb}$, and rbc counts in $\mathrm{P} 8$; Vh/fl/fl $E p o^{f / f l}$ Arnt ${ }^{f l / f l}$ mutant mice and Cre control mice $(n=4-5)$. Lower panel shows relative Epo mRNA expression levels in kidney homogenates from P8;Vh/fl/fl Epo ${ }^{f / f l}, \mathrm{P} 8 ;\left.V h\right|^{f / / f l} E p 0^{f l / f l} A r n t^{f l / f l}$, and control mice $(n=4,4$, and 7, respectively). (B) Shown are $\mathrm{Hct}, \mathrm{Hb}$, and rbc counts for individual 6- to 8-week-old Pepck-Cre Vhff/ffl, Pepck-Cre Vh/ fl/fl Epo ${ }^{f / f l}$, and Cre- control mice ( $n=4,6$, and 7, respectively). (C) Hct, Hb, and rbc counts in 6- to 8-week-old Thp-Cre Vh/fl/fl, Hoxb7-Cre Vh/flfl , and Cre- control mice $\left(n=5,7\right.$, and 5, respectively). ${ }^{* *} P<0.01$; ${ }^{* *} P<0.001$, unpaired 2-tailed Student's $t$ test (A, upper panel); 1-way ANOVA followed by Dunnett's post hoc analysis (A, lower panel, $\mathbf{B}$, and $\mathbf{C})$. Shown are mean values \pm SEM.

$(n=7)$ mice revealed no differences in $\mathrm{Hct}, \mathrm{Hb}$, or rbc values compared with Cre-negative littermate controls $(n=5)$ (Figure 7C), suggesting that $V h l$ ablation in proximal renal epithelium alone is sufficient for the suppression of renal EPO production. In summary, our data establish that the development of anemia in P8; $V h l^{t / f l}$ $E p o^{f l / f l}$ mice was HIF dependent and suggest that HIF activation via $V h l$ gene deletion in the proximal nephron modulates renal EPO production (Figure 8).

\section{Discussion}

In the current study, we utilized inducible and cell type-specific Cre recombinase-mediated gene targeting to investigate the role of non-EPO-synthesizing cell types in the regulation of EPO production in the kidney. We demonstrate that HIF activation in renal epithelial cells via acute $V h l$ gene deletion suppressed erythropoiesis by restricting the number of interstitial fibroblast-like cells that are able to convert to REPCs. This effect localized to the proximal nephron and was associated with renal gene expression and functional changes that indicated a shift from oxidative phosphorylation toward glycolysis as well as decreased $\mathrm{O}_{2}$ consumption due to reduced mitochondrial mass. As a result of epithelial HIF acti- vation, renal cortical $\mathrm{PtO}_{2}$ was elevated despite the presence of marked anemia. Our data suggest that HIF-regulated tubulointerstitial intercellular crosstalk suppresses EPO production under conditions of sustained HIF activation in the kidney. We propose a model whereby HIF activation in a renal cell type that does not synthesize EPO modulates renal EPO production by regulating the number of REPCs.

Ascent to high altitude triggers a pronounced systemic hypoxia response, which results in increased renal EPO synthesis and rbc production. Interestingly, sEPO levels decline rapidly before significant changes in Hct are noted $(12,13)$. While the reasons for this phenomenon are not clear, increased sequestration of EPO from serum by EPO-expressing cells in the bone marrow has been proposed as a possible underlying mechanism (2). The decline in SEPO levels is, however, paralleled by a reduction in renal Epo mRNA expression pointing toward intercellular crosstalk that is directed at local EPO synthesis (24). While it is possible that the hypoxic induction of PHD3 might reduce HIF levels and suppress Epo transcription in REPCs, there is currently no experimental evidence that PHD3 alone regulates renal EPO synthesis (25-29). Findings from our genetic studies, however, would lend experimental sup- 

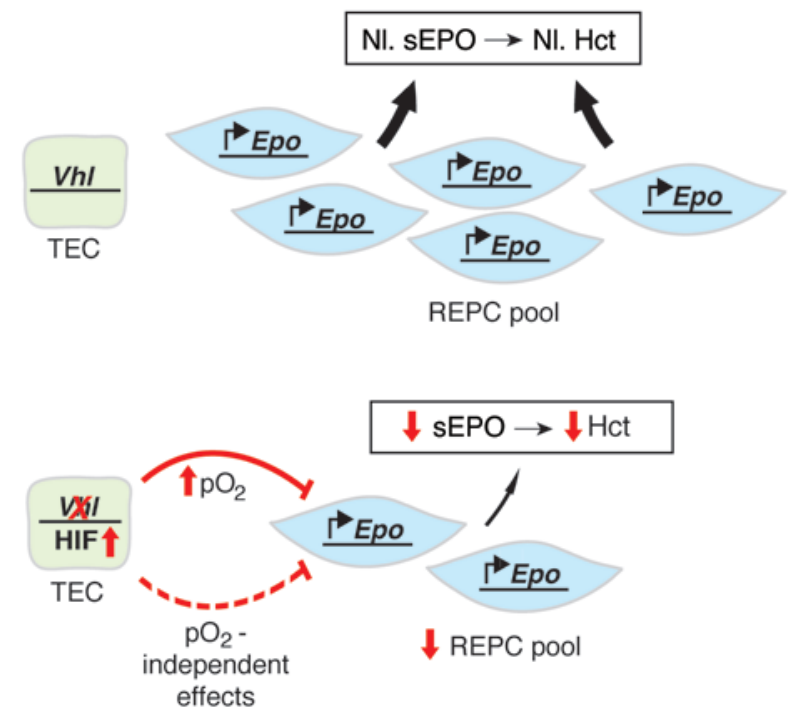

Figure 8. Renal epithelial cells regulate erythropoiesis. Model depicting the role of renal tubular epithelial cells in the regulation of EPO production at baseline (upper panel) and under conditions of constitutive HIF activation as a result of $\mathrm{Vhl}$ inactivation (lower panel). Constitutive HIF stabilization in renal tubular epithelial cells inhibits the conversion of non-EPO-producing peritubular interstitial cells to REPCs.

port to the notion that HIF-regulated metabolic adaptation of renal tubular epithelial cells to hypoxia suppresses EPO synthesis in the kidney partly via modulation of renal $\mathrm{O}_{2}$ consumption.

In our model, both epithelial HIF-1 and HIF-2 were involved in modulating renal EPO production at baseline and under conditions of anemic hypoxia, as concomitant deletion of either Hifla or Hif2a in P8; $\mathrm{Vh}^{f / / l} E \mathrm{Ep}^{\text {fl/l }}$ mice did not restore erythropoiesis to control levels and resulted in inadequate sEPO levels for the degree of anemia observed in these mutants. While epithelial HIF activation in P8; $V h l^{l / l l} E p o^{f / l l}$ mice was associated with anemia, we did not find that inactivation of either epithelial HIF-1 $\alpha$ or HIF-2 $\alpha$ alone (P8;Hifla $\left.a^{\text {f/ll }}, \mathrm{P} 8 ; H i f 2 a^{A / f l}\right)$ or together (P8;Hiflat/fl Hif $\left.2 a^{f / f}\right)$ enhanced renal EPO responses when mice were exposed to prolonged hypoxia ( $8 \% \mathrm{O}_{2}$ for 6 days) or phlebotomy (data not shown). This may be due to the degree of hypoxia generated in this model, i.e., levels of HIF- $\alpha$ stabilization, hypoxic adaptation over time, or timing of the analysis. Our model of nonhypoxic HIF activation is based on Vhl gene deletion in renal epithelial cells alone, which leads to the complete loss of the ability to degrade HIF- $\alpha$ and would mimic severe hypoxia. Under severe hypoxia, factor inhibiting HIF (FIH), an HIF asparagine hydroxylase that hydroxylates an asparagine residue in the $\mathrm{C}$-terminal transactivation domain of HIF- $\alpha$, would further modify HIF-mediated hypoxia responses, as it operates as a second hypoxic switch that regulates recruitment of coactivators to the HIF transcriptional complex (5). Nevertheless, our model provides genetic evidence for HIF-regulated tubulointerstitial cell interaction affecting the conversion of interstitial cells into EPO-producing cells. It is plausible that in the hypoxic kidney, additional nonepithelial cell-derived paracrine signals that also modulate the renal EPO response may come into play.

EPO synthesis occurs in peritubular interstitial fibroblast-like cells, which are normally found in the inner cortex and corticome- dullary region $(7,30)$. The main stimulus for renal EPO production is hypoxia, and small changes in renal oxygenation are rapidly translated into increased $\mathrm{EPO}$ synthesis. $\mathrm{PtO}_{2}$ is therefore a critical determinant of renal EPO output. The regulation of renal $\mathrm{PtO}_{2}$ is complex and depends largely on arterial $\mathrm{O}_{2}$ content, on GFR-driven tubular work load, which in turn determines $\mathrm{O}_{2}$ consumption, and on the degree of arteriovenous $\mathrm{O}_{2}$ shunting, which is a specific feature of the renal microenvironment that results from the counter current exchange of $\mathrm{O}_{2}$ between arterial to venous vessels that are arranged in parallel $(31,32)$. Since GFR and RBF change in parallel under most conditions, the boost in arterial $\mathrm{O}_{2}$ delivery that normally associates with a rise in RBF is largely offset by an increase in $\mathrm{O}_{2}$ consumption, thus limiting its impact on $\mathrm{PtO}_{2}$. As a result, regional $\mathrm{PtO}_{2}$ stays within a relatively narrow range and is highly susceptible to changes in intrarenal metabolism $(3,31)$. HIF activation is known to have profound effects on cellular metabolism. This occurs at multiple levels and includes the increased expression of glucose transporters and glycolytic enzymes, the regulation of TCA cycle flux, and fatty acid oxidation and storage as well as mitochondrial biogenesis, respiration, and autophagy, which could explain the rapid decrease in mitochondrial mass and $\mathrm{O}_{2}$ consumption in our model (17).

We used genetic means to further dissect the contribution of individual nephron segments to the regulation of erythropoiesis and identified the proximal tubule as the nephron segment that modulates EPO production in the kidney. Our observations are in line with previously published physiologic studies that used acetazolamide to block tubular sodium reabsorption in the rat renal proximal tubule. This resulted in a dose-dependent reduction of sEPO in response to hypoxia, possibly, although not measured in this report, through a decrease in renal $\mathrm{O}_{2}$ consumption (11). Consistent with these findings are also studies in humans, which demonstrated that sEPO levels diminish when climbers, who ascend to high altitude, are treated with acetazolamide (33).

The effects of epithelial HIF activation on erythropoiesis were already clearly detectable by day 10 of doxycycline treatment (data not shown). When considering the average mouse erythrocyte life span of approximately 40 days (34), the reduction in rbc numbers by approximately $35 \%$ within 2 weeks after initiation of doxycycline treatment indicates that the suppression of renal Epo transcription must have occurred rapidly. While the timing of EPO suppression is consistent with functional changes in renal metabolism that alter $\mathrm{PtO}_{2}$, our findings also indicate that $\mathrm{PtO}_{2}-$ independent mechanisms may have participated in the modulation of renal EPO synthesis, as the renal Epo response in P8; Vhth/fl $E p o^{f / f l}$ mice remained blunted in response to systemic pharmacologic HIF prolyl-4-hydroxylase inhibition, which was predicted to robustly inhibit HIF PHDs and induce Epo in peritubular interstitial fibroblast-like cells irrespective of $\mathrm{PtO}_{2}$. The mechanisms underlying this observation are unclear and warrant further investigation.

Since our model of pan-epithelial HIF activation is based on $V h l$ gene deletion, it is likely that tubulointerstitial cellular crosstalk comes into play during the pathogenesis of VHL-associated renal cell cancer (RCC) and negatively affects the ability of surrounding peritubular interstitial fibroblast-like cells to synthesize EPO. In contrast with our model of pan-epithelial Vhl inactivation, these effects, however, may not become clinically apparent in 
human RCC patients, as tumorigenesis occurs in a localized fashion and involves only a small fraction of renal epithelial cells.

Renal EPO output correlates with the number of REPCs (6, 7), i.e., the degree of decrease in $\mathrm{PtO}_{2}$ determines the number of peritubular interstitial fibroblast-like cells that switch from a nonEPO-producing to an EPO-producing state. This kind of functional plasticity is not uncommon in the kidney and has been observed in other renal cell types. For example, afferent arteriolar perivascular cells change their functional phenotype and begin to produce renin in response to sodium depletion (35). While $\mathrm{PtO}_{2}$ is a major determinant of REPC pool size, the regulation of REPC plasticity, especially the quality of intra- and extracellular signals that control the ability of interstitial fibroblast-like cells to switch to an EPOproducing state, is only incompletely understood. Myofibroblast transdifferentiation of renal interstitial fibroblast-like cells, which is associated with the development of renal fibrosis and increased extracellular matrix production, has been suggested as suppressing their EPO-producing potential, possibly through activation of $\mathrm{NF}-\kappa \mathrm{B}$ signaling (36). Here, we demonstrate that HIF activation in non-EPO-producing renal epithelial cells modulates REPC pool size and therefore may affect erythropoiesis under kidney injury conditions. This may be particularly important in chronic injury conditions where epithelial HIF stabilization has been shown to associate with the development of fibrosis (37).

Aside from renal interstitial cells, multiple other cell types have been shown to be capable of synthesizing EPO or of modulating erythropoiesis under certain experimental conditions (3). HIF stabilization induced by conditional ablation of $V h l$ or Phds in hepatocytes, astrocytes, renin-producing cells, osteoblasts, and other cell types led to increased sEPO levels and polycythemia through direct activation of tissue-specific Epo transcription (3). In contrast, HIF stabilization in non-EPO-producing keratinocytes causes polycythemia indirectly by redirecting blood flow to the skin, away from kidney and liver, which induced hypoxia and stimulated renal and hepatic EPO production (38). Our data indicate that renal epithelial HIF modulates EPO synthesis in peritubular interstitial fibroblast-like cells, thus providing evidence for kidney-specific non-cell autonomous control of EPO synthesis and suppression of EPO production in the context of HIF activation.

In summary, our genetic studies demonstrate that HIF activation in proximal tubular epithelial cells, which do not synthesize EPO, negatively modulates erythropoiesis. While more experimental studies are needed to further explore the functional relationships between epithelial cells and interstitial fibroblast-like cells in the kidney, our data provide a molecular basis for the participation of renal epithelial cells in the regulation of interstitial cell plasticity and pathogenesis of renal anemia.

\section{Methods}

Generation of mice and genotyping. The generation and genotyping of Vhl, Epo, Arnt, Hifla, and Hif2a conditional alleles and the lacZ ROSA26 reporter (R26R) mouse line as well as LC-1, Pax8-rtTA, Pepck-Cre, Thp-Cre, Hoxb7-Cre, and Pax3-Cre transgenic lines have been described elsewhere (14, 39-47).

Inducible Cre-mediated renal tubular ablation of Vhl, Arnt, Hifla, Hif2a, and/or Epo was achieved by generating mice that carried both the rtTA-dependent Cre-recombinase (LC-1) transgene and expressed rtTA under control of the Pax 8 promoter (Pax8-rtTA). The following genotypes were generated: (a) Vhl/f/ll Pax8-rtTA LC-1, (b)

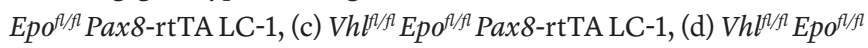
Arnt ${ }^{f / f l}$ Pax8-rtTA LC-1, (e) Vhll/fll Epo ${ }^{f / f l}$ Hifla $a^{f / f l}$ Pax8-rtTA LC-1, and (f) $V h l^{f / f l} \mathrm{Epo}^{f / f l}$ Hif $2 a^{f / f l}$ Pax8-rtTA LC-1. After completion of doxycycline treatment, these were referred to as $\mathrm{P} 8 ; V h l^{f^{l / f l}}, \mathrm{P} 8 ; E p 0^{f / / f l}, \mathrm{P} 8 ; V h l^{f / / f l}$

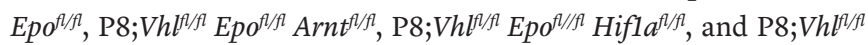
$E p o^{f / f l}$ Hif $2 a^{f / f l}$, respectively. For the activation of the Pax8-rtTA/LC1 conditional knockout system, 4-week-old mice were treated for 2 weeks with doxycycline (Sigma-Aldrich) contained in drinking water at a concentration of $0.2 \mathrm{mg} / \mathrm{ml}$ in $5 \%$ sucrose water.

Constitutive conditional inactivation of $V h l$ and/or Epo in specific nephron segments was achieved by generating mice that were homozygous for the $V h l$ and/or Epo conditional alleles and expressed either a proximal tubule-specific Cre transgene (Pepck-Cre), a distal tubulespecific Cre transgene (Thp-Cre), or a collecting duct-specific Cre transgene (Hoxb7-Cre). Analysis of nephron segment-specific knockout mice was performed at age 6 to 8 weeks. Renal interstitial fibroblast-like cells and tubular epithelial cells were targeted with the Pax3-Cre transgene. The following genotypes were generated: (a) Pepck-Cre Vhlt/fl,

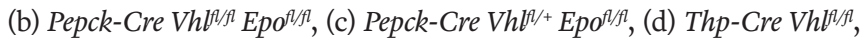

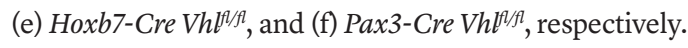

DNA, RNA, and protein analysis. DNA and RNA isolation were performed as described previously (48). For real-time PCR analysis, $1 \mu \mathrm{l}$ of cDNA was subjected to PCR amplification on an ABI StepOnePlus platform using either SYBR green or TaqMan Universal PCR Master Mix (Applied Biosystems). Relative mRNA expression levels were quantified with the relative standard curve method according to the manufacturer's instructions (Applied Biosystems). Primer sequences for the analysis of Epo, Kim-1, Lcn2, F4/80, and Tnfa expression have been published elsewhere $(48,49)$. For the analysis of $I l 1 b$ expression, the following primers were used: fwd, 5'-GGTCAAAGGTTTGGAAGCAG-3'; rev, 5'-TGTGAAATGCCACCTTTTGA-3'. All genes were normalized to $18 \mathrm{~S}$ levels using the $18 \mathrm{~S}$ TaqMan set from Applied Biosystems. Microarray analysis was carried out using an Affymetrix Exon/Gene (WT) microarray chip. Intensity values from each hybridization were scaled versus a global average signal from the same array and normalized by robust multichip average (RMA) analysis. Statistical analysis was performed using a moderated $t$ test and Bonferroni's familywise error rate (FWER) multiple testing. Differentially regulated genes were defined by a 1.5 -fold difference in mean expression values and an adjusted $P$ value of less than 0.05 between groups. Heat maps were created using Heatmap Builder software (http://ashleylab.stanford.edu/tools/tools-scripts.html). All original microarray data were deposited in the NCBI's Gene Expression Omnibus (GEO GSE54172). For RNA in situ detection using the RNAscope Muliplex Fluorescent Assay Kit, paraffin sections of mouse kidneys (5- $\mu \mathrm{m}$ thickness) were processed according to the manufacturer's protocol (Advanced Cell Diagnostics). The hybridized sections were imaged with the Ariol SL-50 automated slide scanner. Nuclear protein extracts for Western blot analysis were prepared as previously described; HIF- $1 \alpha$ was detected with a polyclonal antibody from Novus Biologicals (catalog NB100-449), and HIF- $2 \alpha$ was detected with a custom-made polyclonal antibody from Invitrogen, as previously described (15).

Pharmacological HIF activation. For pharmacological HIF activation, prolyl-4-hydroxylase inhibitor GSK1002083A (GlaxoSmith- 
Kline) was dissolved in $1 \%$ methylcellulose and administered by oral gavage at a dose of $60 \mathrm{mg} / \mathrm{kg}$ twice, 24 and 4 hours prior to tissue harvest (4). Methylcellulose without compound served as control.

Blood work and assessment of renal function. Hcts were determined by microcapillary tube centrifugation in a Micro Hematocrit Centrifuge (International Equipment Co.) or with a Hemavet 950 analyzer (Drew Scientific). sEPO levels were determined by ELISA (R\&D Systems). Reticulocyte counts were measured by FACS analysis of whole blood stained with thiazole orange (Sigma-Aldrich). BUN levels were determined using the QuantiChrom Urea Assay Kit (BioAssay Systems), and serum $\mathrm{Na}^{+}$and $\mathrm{K}^{+}$concentrations were determined with an iStat analyzer and $\mathrm{EC}^{+}$cartridges (Abaxis). For determination of $\mathrm{SaO}_{2}$ and $\mathrm{PaO}_{2}$, blood samples were analyzed with iStat analyzer and $\mathrm{CG}^{+}$cartridges (Abaxis). GFR was assessed using the FITC-inulin single-injection method as previously described (50). GFR was calculated based on a 2-compartment model using GraphPad Prism (GraphPad Software). RBF was estimated by ${ }^{99 \mathrm{~m}} \mathrm{Tc}-\mathrm{MAG} 3$ dynamic scintigraphy as previously described (51).

Histological analysis. For HIF-1 $\alpha$ and HIF- $2 \alpha$ immunohistochemistry, polyclonal rabbit anti-HIF-1 $\alpha$ antibody (Cayman Chemical, catalog 10006421) and polyclonal rabbit anti-HIF- $2 \alpha$ anti-serum PM8 (52) were used at dilutions of 1:10,000 and 1:20,000, respectively, and detected with the CSA-II High Signal Amplification Kit and the Rabbit Link Reagent (Dako). For detection of CD45 leukocytes, anti-mouse monoclonal anti-CD45 antibody (BD Biosciences - Pharmingen, catalog 550539) was used and detected with the Vectastain Elite Kit (Vector). X-gal staining was performed on frozen tissues sections according to MacGregor (53).

High-resolution respirometry. Respiratory activities in tissue samples from cortex and medulla of mutant and control mice were analyzed with the O2k Oxygraph System (Oroboros). Tissue samples from the cortex and medulla were dissected, minced on ice, and placed on ice in mitochondrial respiration buffer (final concentration: $110 \mathrm{mM}$ sucrose, $60 \mathrm{mM}$ K-lactobionate, $0.5 \mathrm{mM}$ EGTA, $1 \mathrm{~g} / \mathrm{l}$ BSA essentially fatty acid free, $3 \mathrm{mM} \mathrm{MgCl} 2,20 \mathrm{mM}$ taurine, $10 \mathrm{mM} \mathrm{KH} 2 \mathrm{PO} 4,20 \mathrm{mM}$ Hepes, adjusted to $\mathrm{pH} 7.1$ with $\mathrm{KOH}$ ) at a concentration of $1 \mathrm{mg} / \mathrm{ml}$. Samples from $\mathrm{P} 8 ; V h l^{f l f l} \mathrm{E} p \mathrm{o}^{f / f l}$ and control mice were placed into an $\mathrm{O} 2 \mathrm{k}$ Oxygraph (Oroboros), and $\mathrm{O}_{2}$ consumption and flux were determined for state 2 (presence of substrate in the absence of ADP), cI, cII, and cIV separately by titration of the following substances: (a) glutamate/ malate (final concentration: $10 / 2 \mathrm{mM}$ ), (b) ADP (final concentration: $25 \mathrm{mM}$ ), (c) rotenone (final concentration: $0.5 \mu \mathrm{M}$ ), (d) succinate (final concentration: $10 \mathrm{mM}$ ), (e) antimycin A (final concentration: $2.5 \mu \mathrm{M}$ ), and (f) TMPD/ascorbate (final concentration: 0.5/2 mM). Measurements were live recorded using DatLab software (Oroboros).

Metabolic analysis and cortical $\mathrm{PtO}_{2}$ measurements. CS activity was measured as previously described (21). Briefly, aliquots from samples used for high-resolution respirometry were homogenized, and $20 \mu \mathrm{l}$ of homogenate was added to $180 \mu \mathrm{l}$ medium containing $0.1 \mathrm{mM} \mathrm{5,5-dithio-bis-(2-nitrobenzoic)} \mathrm{acid} \mathrm{(DTNB)} \mathrm{(Sigma-}$ Aldrich), $0.5 \mathrm{mM}$ oxaloacetate (Sigma-Aldrich), 50 M EDTA (Sigma-Aldrich), $0.31 \mathrm{mM}$ acetyl coenzyme A (Sigma-Aldrich), $5 \mathrm{mM}$ triethanolamine hydrochloride (Fluka), and 0.1 M Tris-HCl, pH 8.1 (Sigma-Aldrich). The activity of CS was measured spectrophotometrically at $412 \mathrm{~nm}$. Glucose uptake was measured in renal medulla with 2-DG at $13 \mu \mathrm{Ci} /$ mouse and normalized to brain as previously described (54). Polar metabolites were analyzed as previously described (55). Mice were fasted for 4 hours prior to intravenous injection of $\mathrm{U}^{-13} \mathrm{C}$ glucose ( $\left.20 \mathrm{mg} / \mathrm{mouse}\right)$. At 25 minutes following the injection, mice were sacrificed and kidneys were harvested. ${ }^{13} \mathrm{C}$ isotopic enrichment was analyzed in total kidneys using Agilent 6890/5975N GC-MS equipped with 25 m DB-5ms capillary column for analysis of isotopic enrichment.

For cortical $\mathrm{PtO}_{2}$ measurements, mice were subjected to inhalation anesthesia with isoflurane. Core body temperature was monitored with a rectal probe and maintained between $36.5^{\circ} \mathrm{C}$ and $37.5^{\circ} \mathrm{C}$. The left kidney was exposed through flank incision and was stabilized without causing excessive strain on renal vessels. The kidney capsule was removed, and the left kidney was covered with mineral oil. Renal $\mathrm{PtO}_{2}$ was measured with a modified Clark-type microelectrode $(\emptyset=8-10 \mu \mathrm{m}$, Unisense). Two-point calibration was carried out in water saturated with either $\mathrm{N}_{2}$ gas or ambient air at $37^{\circ} \mathrm{C}$. For every mouse, a minimum of 3 independent measurements were obtained per kidney $1 \mathrm{~mm}$ below the renal surface after a linear correlation between $\mathrm{O}_{2}$ tension and electric current was established. For statistical analysis, only average mean values from individual mice were used.

Statistics. Data reported represent mean values \pm SEM. Statistical analyses were performed with Prism 5.0b software (GraphPad Software). When 2 groups of mice were compared, the unpaired, 2-tailed Student's $t$ test was used; for comparison of 3 and more groups, 1-way ANOVA followed by Dunnett's post hoc analysis was used. $P<0.05$ was considered statistically significant.

Study approval. All procedures involving mice were performed in accordance with NIH guidelines for the use and care of live animals and were approved by the Institutional Animal Care and Use Committee (IACUC) of Vanderbilt University.

\section{Author contributions}

$\mathrm{NMF}$ and VHH conceived and designed the research studies, analyzed and interpreted data, wrote the manuscript, and created the figures. NMF, QL, HK, FS, and OD performed experiments and acquired and analyzed data. TAI, PMO, and JF helped with design and interpretation of research studies or contributed reagents or technical expertise.

\section{Acknowledgments}

This work was supported by the Krick-Brooks Chair in Nephrology (to V.H. Haase) and by NIH grants R01-DK101791, R01DK081646, and R01-DK080821 and a Departments of Veterans Affairs Merit Award (1I01BX002348) (all to V.H. Haase). The authors wish to thank Peter Ratcliff (University of Oxford) for providing the PM8 HIF-2 $\alpha$ antibody, Mark J. Koury and Andres A. Urrutia Elorduy (Vanderbilt University) for their critical reading of the manuscript, the Vanderbilt Institute of Imaging Science for performing the ${ }^{99 \mathrm{~m}} \mathrm{Tc}-\mathrm{MAG} 3$ dynamic scintigraphy, the Vanderbilt Technologies for Advanced Genomics (VANTAGE) core for performing the mRNA expression array analysis, and the Vanderbilt Diabetes Research and Training Center (P30-DK20593) and Vanderbilt Mouse Metabolic Phenotyping Center (DK059637) for support and help with animal work and metabolic analyses.

Address correspondence to: Volker H. Haase, Division of Nephrology and Hypertension, Vanderbilt University Medical Center, C-3119A MCN, 1161 21st Avenue So., Nashville, Tennessee 37232-2372, USA. Phone: 615.343.7254; E-mail: volker.haase@vanderbilt.edu. 
1. Jelkmann W. Molecular biology of erythropoietin. Intern Med. 2004;43(8):649-659.

2. Koury MJ. Erythropoietin: the story of hypoxia and a finely regulated hematopoietic hormone. Exp Hematol. 2005;33(11):1263-1270.

3. Koury MJ, Haase VH. Anaemia in kidney disease: harnessing hypoxia responses for therapy. Nat Rev Nephrol. 2015;11(7):394-410.

4. Kapitsinou PP, et al. Hepatic HIF-2 regulates erythropoietic responses to hypoxia in renal anemia. Blood. 2010;116(16):3039-3048.

5. Kaelin WG Jr, Ratcliffe PJ. Oxygen sensing by metazoans: the central role of the HIF hydroxylase pathway. Mol Cell. 2008;30(4):393-402.

6. Koury ST, Koury MJ, Bondurant MC, Caro J, Graber SE. Quantitation of erythropoietin-producing cells in kidneys of mice by in situ hybridization: correlation with hematocrit, renal erythropoietin mRNA, and serum erythropoietin concentration. Blood. 1989;74(2):645-651.

7. Obara N, et al. Repression via the GATA box is essential for tissue-specific erythropoietin gene expression. Blood. 2008;111(10):5223-5232.

8. Bachmann S, Le Hir M, Eckardt KU. Co-localization of erythropoietin mRNA and ecto-5'nucleotidase immunoreactivity in peritubular cells of rat renal cortex indicates that fibroblasts produce erythropoietin. J Histochem Cytochem. 1993;41(3):335-341.

9. Asada N, et al. Dysfunction of fibroblasts of extrarenal origin underlies renal fibrosis and renal anemia in mice. JClin Invest. 2011;121(10):3981-3990.

10. Jelkmann W. Erythropoietin: structure, control of production, and function. Physiol Rev. 1992;72(2):449-489.

11. Eckardt KU, Kurtz A, Bauer C. Regulation of erythropoietin production is related to proximal tubular function. Am J Physiol. 1989; 256(5 pt 2):F942-F947.

12. Faura J, et al. Effect of altitude on erythropoiesis. Blood. 1969;33(5):668-676.

13. Abbrecht PH, Littell JK. Plasma erythropoietin in men and mice during acclimatization to different altitudes. J Appl Physiol. 1972;32(1):54-58.

14. Traykova-Brauch $\mathrm{M}$, et al. An efficient and versatile system for acute and chronic modulation of renal tubular function in transgenic mice. Nat Med. 2008;14(9):979-984.

15. Rankin EB, et al. Hypoxia-inducible factor-2 (HIF-2) regulates hepatic erythropoietin in vivo. J Clin Invest. 2007;117(4):1068-1077.

16. Mathia S, et al. Action of hypoxia-inducible factor in liver and kidney from mice with Pax8-rtTAbased deletion of von Hippel-Lindau protein. Acta Physiol (Oxf). 2013;207(3):565-576.

17. Semenza GL. Regulation of metabolism by hypoxia-inducible factor 1. Cold Spring Harb Symp Quant Biol. 2011;76:347-353.

18. Kim JW, Tchernyshyov I, Semenza GL, Dang CV. HIF-1-mediated expression of pyruvate dehydrogenase kinase: a metabolic switch required for cellular adaptation to hypoxia. Cell Metab. 2006;3(3):177-185.

19. Papandreou I, Cairns RA, Fontana L, Lim AL, Denko NC. HIF-1 mediates adaptation to hypoxia by actively downregulating mitochondrial oxygen consumption. Cell Metab. 2006;3(3):187-197.
20. Zhang H, et al. HIF-1 inhibits mitochondrial biogenesis and cellular respiration in VHL-deficient renal cell carcinoma by repression of C-MYC activity. Cancer Cell. 2007;11(5):407-420.

21. Kuznetsov AV, et al. Evaluation of mitochondrial respiratory function in small biopsies of liver. Anal Biochem. 2002;305(2):186-194.

22. Artunc F, Risler T. Serum erythropoietin concentrations and responses to anaemia in patients with or without chronic kidney disease. Nephrol Dial Transplant. 2007;22(10):2900-2908.

23. Rankin EB, Tomaszewski JE, Haase VH. Renal cyst development in mice with conditional inactivation of the von Hippel-Lindau tumor suppressor. Cancer Res. 2006;66(5):2576-2583.

24. Eckardt KU, Dittmer J, Neumann R, Bauer C, Kurtz A. Decline of erythropoietin formation at continuous hypoxia is not due to feedback inhibition. Am J Physiol. 1990;258(5 pt 2):F1432-F1437.

25. Takeda K, et al. Regulation of adult erythropoiesis by prolyl hydroxylase domain proteins. Blood. 2008;111(6):3229-3235.

26. Minamishima YA, et al. A feedback loop involving the Phd3 prolyl hydroxylase tunes the mammalian hypoxic response in vivo. Mol Cell Biol. 2009;29(21):5729-5741.

27. Minamishima YA, Kaelin WG Jr. Reactivation of hepatic EPO synthesis in mice after PHD loss. Science. 2010;329(5990):407.

28. Laitala A, et al. Transmembrane prolyl 4-hydroxylase is a fourth prolyl 4-hydroxylase regulating EPO production and erythropoiesis. Blood. 2012;120(16):3336-3344.

29. Querbes W, et al. Treatment of erythropoietin deficiency in mice with systemically administered siRNA. Blood. 2012;120(9):1916-1922.

30. Koury ST, Bondurant MC, Koury MJ. Localization of erythropoietin synthesizing cells in murine kidneys by in situ hybridization. Blood. 1988;71(2):524-527.

31. Evans RG, Gardiner BS, Smith DW, O'Connor PM. Intrarenal oxygenation: unique challenges and the biophysical basis of homeostasis. Am J Physiol Renal Physiol. 2008;295(5):F1259-F1270.

32. Haase VH. Mechanisms of hypoxia responses in renal tissue. J Am Soc Nephrol. 2013;24(4):537-541.

33. Reinhart WH, Goerre S, Bärtsch P. Acetazolamide reduces the erythropoietin response to hypoxia at high altitude in humans. JWilderness Med.1994;5(3):312-317.

34. Van Putten LM. The life span of red cells in the rat and the mouse as determined by labeling with DFP32 in vivo. Blood. 1958;13(8):789-794.

35. Sequeira Lopez ML, Pentz ES, Nomasa T, Smithies O, Gomez RA. Renin cells are precursors for multiple cell types that switch to the renin phenotype when homeostasis is threatened. Dev Cell. 2004;6(5):719-728.

36. Souma T, et al. Plasticity of renal erythropoietinproducing cells governs fibrosis. J Am Soc Nephrol. 2013;24(10):1599-1616.

37. Higgins DF, et al. Hypoxia promotes fibrogenesis in vivo via HIF-1 stimulation of epithelialto-mesenchymal transition. JClin Invest. 2007;117(12):3810-3820.

38. Boutin AT, et al. Epidermal sensing of oxygen is essential for systemic hypoxic response. Cell.
2008;133(2):223-234

39. Soriano P. Generalized lacZ expression with the ROSA26 Cre reporter strain. Nat Genet. 1999;21(1):70-71.

40. Li J, Chen F, Epstein JA. Neural crest expression of Cre recombinase directed by the proximal Pax3 promoter in transgenic mice. Genesis. 2000;26(2):162-164.

41. Ryan HE, et al. Hypoxia-inducible factor-1 $\alpha$ is a positive factor in solid tumor growth. Cancer Res. 2000;60(15):4010-4015.

42. Haase VH, Glickman JN, Socolovsky M, Jaenisch R. Vascular tumors in livers with targeted inactivation of the von Hippel-Lindau tumor suppressor. Proc Natl Acad Sci U S A. 2001;98(4):1583-1588

43. Schonig K, Schwenk F, Rajewsky K, Bujard H. Stringent doxycycline dependent control of CRE recombinase in vivo. Nucleic Acids Res. 2002;30(23):e134.

44. Yu J, Carroll TJ, McMahon AP. Sonic hedgehog regulates proliferation and differentiation of mesenchymal cells in the mouse metanephric kidney. Development. 2002;129(22):5301-5312.

45. Stricklett PK, Taylor D, Nelson RD, Kohan DE. Thick ascending limb-specific expression of Cre recombinase. Am J Physiol Renal Physiol. 2003;285(1):F33-F39.

46. Walisser JA, Bunger MK, Glover E, Harstad EB, Bradfield CA. Patent ductus venosus and dioxin resistance in mice harboring a hypomorphic Arnt allele. J Biol Chem. 2004;279(16):16326-16331.

47. Zeigler BM, Vajdos J, Qin W, Loverro L, Niss K. A mouse model for an erythropoietin-deficiency anemia. Dis Model Mech. 2011;3(11-12):763-772.

48. Liu Q, Davidoff O, Niss K, Haase VH. Hypoxiainducible factor regulates hepcidin via erythropoietin-induced erythropoiesis. JClin Invest. 2012;122(12):4635-4644.

49. Kobayashi H, et al. Myeloid cell-derived hypoxiainducible factor attenuates inflammation in unilateral ureteral obstruction-induced kidney injury. JImmunol. 2012;188(10):5106-5115.

50. Qi Z, et al. Serial determination of glomerular filtration rate in conscious mice using FITCinulin clearance. Am J Physiol Renal Physiol. 2004;286(3):F590-F596.

51. Tantawy MN, et al. Assessment of renal function in mice with unilateral ureteral obstruction using 99mTc-MAG3 dynamic scintigraphy. BMC Nephrol. 2012;13:168.

52. Wiesener MS, et al. Widespread hypoxiainducible expression of HIF-2alpha in distinct cell populations of different organs. FASEB J. 2003;17(2):271-273.

53. MacGregor GR, Zambrowicz BP, Soriano P. Tissue non-specific alkaline phosphatase is expressed in both embryonic and extraembryonic lineages during mouse embryogenesis but is not required for migration of primordial germ cells. Development. 1995;121(5):1487-1496.

54. Ayala JE, et al. Chronic treatment with sildenafil improves energy balance and insulin action in high fat-fed conscious mice. Diabetes. 2007;56(4):1025-1033.

55. Noguchi $Y$, et al. Tracking cellular metabolomics in lipoapoptosis- and steatosis-developing liver cells. Mol Biosyst. 2011;7(5):1409-1419. 